\title{
Singular perturbations with boundary conditions and the Casimir effect in the half space
}

\author{
S. Albeverio(1), G. Cognola ${ }^{(2)}$, M. Spreafico ${ }^{(3)}$, S. Zerbini ${ }^{(2)}$ \\ (1) Inst. Appl. Math., HCM, IZKS, SFB611 University of Bonn, Wegelerstr. 6, 53115 Bonn, Germany \\ and BiBoS (Bielefeld-Bonn), CERFIM (Locarno); Accademia di Architettura (USI, Mendrisio); \\ Dipartimento di Matematica, Università di Trento. \\ (2) Dipartimento di Fisica, Università di Trento, \\ Povo 38100, Italy, and INFN, Gruppo Collegato di Trento. \\ (3) Dipartimento di Matematica, Università di Trento, Povo 38100, Italy. \\ On leave from ICMC, Universidade de São Paulo, São Carlos, Brasil.
}

\begin{abstract}
We study the self adjoint extensions of a class of non maximal multiplication operators with boundary conditions. We show that these extensions correspond to singular rank one perturbations (in the sense of [3]) of the Laplace operator, namely the formal Laplacian with a singular delta potential, on the half space. This construction is the appropriate setting to describe the Casimir effect related to a massless scalar field in the flat space time with an infinite conducting plate and in the presence of a point like "impurity". We use the relative zeta determinant (as defined in [24] and [30]) in order to regularize the partition function of this model. We study the analytic extension of the associated relative zeta function, and we present explicit results for the partition function, and for the Casimir force.
\end{abstract}

2000 Mathematics Subject Classification: 47F05, 81Q10, 58J52.

Keywords: singular perturbations, boundary conditions; Casimir effect, delta potentials, point interactions; finite temperature, quantum fields; self-adjoint extensions, functional determinants, zeta-function renormalization.

\section{INTRODUCTION}

Recently, there has been a growing interest in the Casimir effect, namely the manifestation of vacuum energy at experimental as well as at theoretical level (see, for example [22, 23] and references therein). Because of the increasing interest in the Casimir effect and in spite of several results which have already been obtained, "a solvable model" that permits to obtain systematically explicit results is of the greatest interest. In this paper we present such a model. Moreover we put at work mathematical techniques, which are of interest by themselves.

We shall study the Casimir effect related to a massless scalar field in a flat space-time modified by the presence of a pointlike (uncharged) "impurity", modelled by delta-like potentials, in manifolds with and without boundary.

The boundaryless delta potential case has been already treated (see for example [2, 8, 21, 26, 28, 29]. This case is also referred to as semi-transparent boundary conditions (see [7, 16, 17, 20] and references therein). Here we shall deal with the new case of a delta potential on the half space.

On general grounds, from one side, we present a rigorous mathematical description of the Schrödinger-like operators with delta-like potentials, and from the other side, we will make use of a technique to regularize the functional determinant of self-adjoint elliptic operators defined on non compact manifolds associated with continuous spectrum.

In order to start formulating the problem, we use the approach of Finite Temperature Quantum Field Theory based on the imaginary time formalism (see, for example [15] [10], and [9, 11, 12] [25], and references therein). We consider a massless scalar free field in four dimensional Minkowski space-time interacting with an external field represented by a potential $V$. Thus, one is dealing with the manifold $X(T)=S_{\beta / 2 \pi}^{1} \times M$, where $S_{r}^{1}$ is the circle of radius $r, \beta=\frac{1}{T}$, the period of the imaginary compactified time, is the inverse of the temperature, and $M$ is a three dimensional manifold. The relevant operator reads $H=-\Delta_{X(T)}+V=-\partial_{u}^{2}-\Delta_{M}+V=-\partial_{u}^{2}+L_{M}$, where $\Delta_{Y}$ is the Laplace-Beltrami operator on a manifold $Y$ defined by some Riemannian structure, and $V: M \rightarrow \mathbb{R}$ is a suitable potential.

The canonical partition function at temperature $T$ of this model may be formally written as

$$
\log Z=-\frac{\beta}{2} \sum_{\lambda \in \operatorname{Sp} L_{M}} \lambda^{1 / 2}-\sum_{\lambda \in \operatorname{Sp} L_{M}}\left(1-\mathrm{e}^{-\beta \sqrt{\lambda}}\right),
$$

here $\operatorname{Sp} L_{M}$ is the spectrum (a self-realization of) $L_{M}$. We are assuming $M$ to be a compact manifold and $V$ a smooth potential. The first term on the right-hand side of equation (11) corresponds to the vacuum energy contribution (Casimir energy), given by

$$
E_{c}=-\lim _{\beta \rightarrow \infty} \partial_{\beta} \log Z=\frac{1}{2} \sum_{\lambda \in \operatorname{Sp} L_{M}} \lambda^{1 / 2}
$$


while the second one, corresponding to the statistical sum contribution, is vanishing in the zero temperature limit. In order to give a meaning to the divergent first term in equation (1), one may make use of the well-known zeta function regularization, namely one introduces the generalized zeta function, defined for large values of the real part of $s$ by

$$
\zeta\left(s ; L_{M}\right)=\sum_{\lambda \in \operatorname{Sp} L_{M}} \lambda^{-s}
$$

and by analytic continuation elsewhere, and one replaces equation (2) by $E_{c}=\frac{1}{2} \zeta\left(-1 / 2 ; L_{M}\right)$.

Nevertheless this approach does not work in general, because it may happen that $\zeta\left(s ; L_{M}\right)$ is $\operatorname{singular}$ in $s=-1 / 2$. A possible approach is to consider $\log Z$ as a regularized functional determinant of the operator $H=-\partial_{u}^{2}+L_{M}$, namely $\log Z=-\frac{1}{2} \zeta^{\prime}(0, H)$ (see, for example, [14]). As a consequence, it is possible to show that it can be expressed in terms of some invariants of the geometric zeta function, i.e. the zeta function of the restriction of $H$ to $M$, and introducing another spectral function, the generalized Dedekind eta function [25], defined for a positive operator $A$ with discrete spectrum by

$$
\eta(\tau ; A)=\prod_{\lambda \in \sigma(A)}\left(1-\mathrm{e}^{-\tau \sqrt{\lambda}}\right)
$$

In fact, assuming that $-\Delta_{M}+V$ has trivial kernel, by Proposition 3 of [25] (see also [9, 11, 14]), we have

$$
\zeta(0 ; H)=\frac{1}{T} \operatorname{Res}_{s=-\frac{1}{2}} \zeta\left(s ;-\Delta_{M}+V\right)
$$

while by Corollary 1 of 25$]$

$$
\zeta^{\prime}(0 ; H)=-\frac{1}{2 T} \operatorname{Res}_{s=-\frac{1}{2}} \zeta\left(s ;-\Delta_{M}+V\right)-\frac{1-\log 2}{T} \operatorname{Res}_{s=-\frac{1}{2}} \zeta\left(s ;-\Delta_{M}+V\right)-2 \log \eta\left(\frac{1}{T} ;-\Delta_{M}+V\right)
$$

where, for a meromorphic function $f(s), \operatorname{Res}_{0}, \operatorname{Res}_{\mathrm{k}}$ are defined by means of the Laurent expansion

$$
f(s)=\operatorname{Res}_{\substack{s=s_{0} \\ 0}} f(s)+\sum_{k=1}^{\infty} \frac{1}{\left(s-s_{0}\right)^{k}} \operatorname{Res}_{\substack{\mathrm{k} \\ s=s_{0}}} f(s) .
$$

In this paper, we will consider explicitly differential operators with a singular potential on non compact manifolds with or without boundary. Thus we shall need to generalize the above results to the non compact case. This will be done in details in the next sections.

Now let us introduce the class of models we are going to investigate. In order to describe the class of operators we shall deal with, we start with an heuristic treatment which will be mathematically justified in the next sections. We first recall the Lipmann-Schwinger equations for an operator $H=H_{0}+V$ defined in $L_{2}\left(\mathbb{R}^{n}\right)$, where $H_{0}=-\Delta$ is minus Laplacian and $V$ is a suitable non confining potential. They are given by

$$
\Psi^{ \pm}(x)=\Psi_{0}^{ \pm}(x)+\int_{\mathbb{R}^{n}} G_{k}^{(0)}(x, y) V(y) \Psi^{ \pm}(y) d y, \quad x \in \mathbb{R}^{n}
$$

where $G_{k}^{(0)}(x, y)$ is the Green function of the unperturbed operator $H_{0}$, namely

$$
\left(H_{0}-k^{2}\right) G_{k}^{(0)}(x, y)=\delta(x-y)
$$

For example, for $n=1$, one has

$$
G_{k}^{(0)}(x, y)=\frac{i}{2 k} e^{i k|x-y|}
$$

The above integral equation is the counterpart of the well known resolvent identity associated with the resolvent of the operator $H$.

We shall consider singular perturbations of the form

$$
V(x)=g \delta(x-a)
$$


where $g$ is the real coupling constant, and we limit our analysis to the cases $n=1,2,3$, since only within these cases, one may implement delta-like interactions by self-adjoint operators in Hilbert space (see, for example [1]). Hence, heuristically

$$
H=-\Delta+g \delta(x-a)
$$

In this case, one formally has as solution

$$
\Psi^{ \pm}(x)=\Psi_{0}^{ \pm}(x)+g G_{k}^{(0)}(x, a) \Psi^{ \pm}(a) .
$$

Since $G_{k}^{(0)}(x, a)$ is singular when $x \rightarrow a$ for $n=2,3$, the above solution of the original integral equation is inconsistent and one has to deal with a regularization and a renormalization procedure, first introduced in [5]. Here we describe a regularization in the configuration space. First, the regularization may be achieved by making the replacement $g \rightarrow g(\varepsilon)$ and $G_{k}^{(0)}(x, a) \rightarrow G_{k}^{(0)}(x+\varepsilon, a)$, for $y>0$. As a result, neglecting terms which vanish as the cutoff $\epsilon$ is removed, i.e. when $\varepsilon \rightarrow 0$, we may solve the above equation and arrive at

$$
\Psi^{ \pm}(a)=\Psi_{0}^{ \pm}(a)+g(\varepsilon) G_{k}^{(0)}(a, a+\varepsilon) \Psi^{ \pm}(a) .
$$

Thus, the regularized solution may be written as

$$
\Psi^{ \pm}(x)=\Psi_{0}^{ \pm}(x)+\frac{1}{\frac{1}{g(\varepsilon)}-G_{k}^{(0)}(a, a+\varepsilon)} G_{k}^{(0)}(x, a) \Psi_{0}^{ \pm}(a)
$$

Furthermore, the renormalization consists in assuming that $g(\varepsilon)$ vanishes in the limit $\varepsilon \rightarrow 0$ in such a way that

$$
\frac{1}{g(\varepsilon)}-G_{k}^{(0)}(a, a+\varepsilon)=\frac{1}{g_{R}}-\operatorname{Res}_{\varepsilon=0} G_{k}^{(0)}(a, a+\varepsilon)+O(\varepsilon),
$$

for some $g_{R} \neq 0$. As a consequence, one may remove the cutoff and one arrives at a finite expression, where renormalized quantities appear, that is

$$
\Psi^{ \pm}(x)=\Psi_{0}^{ \pm}(x)+\frac{1}{\frac{1}{g_{R}}-\operatorname{Res}_{0 z=a} G_{k}^{(0)}(a, z)} G_{k}(x, a) \Psi_{0}^{ \pm}(a) .
$$

For $n>3$, formally the above expression is still valid, but the interpretation of $\Psi^{ \pm}(x)$ as scattering states related to a self-adjoint Hamiltonian defined on an Hilbert space no longer holds.

As an example, let us consider $n=3$ and $a=0$. Then, one has

$$
G_{k}^{(0)}(x)=\frac{1}{4 \pi|x|} e^{i k|x|}
$$

and

$$
\Psi^{ \pm}(x)=e^{ \pm i k x}+\frac{1}{\frac{1}{g_{R}}-i k} \frac{e^{i k|x|}}{|x|}
$$

Instead, for $n=2$, one has

$$
G_{k}(x, y)=\frac{i}{4} H_{0}^{(1)}(k|x-y|)
$$

$H_{0}^{(1)}$ being a Hankel function. Due to the presence of a logarithmic singularity for $x=y$, the regularization procedure leads to the appearance of an arbitrary dimensional scale $\ell$ and the regularized coupling constant has to be "running", in order to ensure the independence of the physical observables from $\ell$. The result is

$$
\Psi^{ \pm}(x)=\Psi_{0}^{ \pm}(x)+\frac{(i / 4) H_{0}^{(1)}(k|x|)}{\frac{1}{g_{R}(\ell)}+\frac{1}{2 \pi}(\ln (k \ell / 2 i)-\Psi(1))}
$$


Coming back to the $n=3$ case, one may obtain the physical meaning of $g_{R}$, considering the non relativistic scattering of a particle of mass $m>0$. In this case, the operator $H_{0}$ is the kinetic energy (the Planck constant being taken to be one) and we have for the scattering wave-functions $\Psi^{ \pm}(x)$

$$
\Psi^{ \pm}(\vec{x})=e^{ \pm i k x}+\frac{2 m g_{R}}{1-2 i m g_{R} k} \frac{e^{i k|x|}}{|x|} .
$$

The scattering amplitude can be read off and is

$$
f(k)=\frac{2 m g_{R}}{1-2 i m g_{R} k},
$$

and the differential cross section is given by

$$
\frac{d \sigma}{d \Omega}=|f(k)|^{2}=\frac{4 m^{2} g_{R}^{2}}{1+4 m^{2} g_{R}^{2} k^{2}} .
$$

The scattering length may be defined as

$$
a^{2}=\lim _{k \rightarrow 0}|f(k)|^{2}
$$

in such a way that $\lim _{k \rightarrow 0} \sigma(k)=4 \pi a^{2}$. Thus,

$$
a^{2}=4 m^{2} g_{R}^{2}
$$

namely the regularized coupling constant is proportional to the scattering length of the related non relativistic 3dimensional scattering process.

It is easy to show that equation (4) is equivalent to the following expression for the kernels of the resolvents

$$
G_{\lambda}(x, y)=G_{\lambda}^{(0)}(x, y)+\frac{1}{\frac{1}{g_{R}}-\operatorname{Res}_{0 z=a} G_{k}^{(0)}(a, z)} G_{\lambda}^{(0)}(x, a) G_{\lambda}^{(0)}(y, a) .
$$

This formula is valid in general. For example, when the unperturbed operator $H_{0}$ is minus the Laplace operator defined in the manifold with boundary $\mathbb{R}^{+} \times \mathbb{R}^{n-1}$, we may repeat the above arguments and arrive at equation (5), in which $G_{\lambda}^{(0)}(x, y)$ and $G_{\lambda}(x, y)$ now satisfy a suitable boundary condition, for example the Dirichlet boundary condition

$$
G_{\lambda}^{(0)}(0, y)=0 .
$$

In the physically relevant case of $n=3$, we have

$$
G_{\lambda}^{(0)}(x, y)=\frac{1}{4 \pi}\left(\frac{e^{i \sqrt{\lambda}|x-y|}}{|x-y|}-\frac{e^{i \sqrt{\lambda}|x-R y|}}{|x-R y|}\right),
$$

where $R$ is the spatial reflection with respect to the plane which forms the boundary $\mathbb{R}^{2}$. For example, in this case, the renormalization leads to

$$
\frac{1}{g(\varepsilon)}-G_{\lambda}^{(0)}(\varepsilon)=\frac{1}{g_{R}}-\frac{\lambda}{4 \pi}-\frac{e^{-2 \lambda a}}{2 a}+O(\varepsilon)
$$

Again, for $n>3$, one may formally consider the above expressions, but without any references to some Hilbert space.

In the following sections, making use of the method of self-adjoint extensions and the general theory of singular perturbations, we will present a rigorous mathematical derivation of the above heuristic results.

\section{SELF ADJOINT EXTENSIONS OF NON MAXIMAL MULTIPLICATION OPERATORS}

\section{A. General setting}

Let $\mathcal{H}$ be an Hilbert space (complete and separable), and $A$ a self adjoint operator in $\mathcal{H}$. Fixing a suitable restriction $\dot{A}$ of $A$, it is possible to construct a one parameter family of self adjoint operators $A_{\alpha}$ containing the initial operator 
$A$. This quite general setting was developed in [3], Section 1.2.2. We recall here the main points of the construction, and we give a new proof of the main result, stated in Lemma 1.2.3 of [3] . Let $|A|=\left(A^{\dagger} A\right)^{\frac{1}{2}}$, and for $s \geq 0$, let

$$
\mathcal{H}_{s}=\operatorname{dom}\left(|A|^{\frac{s}{2}}\right)=\left\{v \in \mathcal{H} \mid(|A|+I)^{\frac{s}{2}} v \in \mathcal{H}\right\} .
$$

Note that $\mathcal{H}_{2}=\operatorname{dom}(A)$ and $\mathcal{H}_{0}=\mathcal{H}$. $\mathcal{H}_{s}$ is a complete Hilbert space with scalar product

$$
(u, v)_{s}=\left((|A|+I)^{s} u, v\right)_{0} .
$$

Obviously, $(|A|+z I)^{\frac{s}{2}}$ is an isometry of $\mathcal{H}_{s}$ onto $\mathcal{H}$ for all $s$ and for all $z$ that is neither zero nor a negative real number. Let $\mathcal{H}_{-s}=\mathcal{H}_{s}^{\dagger}$ be the adjoint space of $\mathcal{H}_{s}$. We define the mapping

$$
\begin{aligned}
& (|\mathcal{A}|+z I)^{\frac{s}{2}}: \mathcal{H} \rightarrow \mathcal{H}_{-s}, \\
& (|\mathcal{A}|+z I)^{\frac{s}{2}}: u \mapsto(|\mathcal{A}|+z I)^{\frac{s}{2}} u,
\end{aligned}
$$

by

$$
\left((|\mathcal{A}|+z I)^{\frac{s}{2}} u\right)(v)=\left(u,(|A|+z I)^{\frac{s}{2}} v\right)_{0},
$$

for all $v \in \mathcal{H}_{s}$, and $\operatorname{Re}(z)>0$. On the other hand, for each $\mathrm{u} \in \mathcal{H}_{-s}$, there exists a vector $u^{\prime} \in \mathcal{H}_{s}$ such that

$$
\mathrm{u}(v)=\left(u^{\prime}, v\right)_{s},
$$

for all $v \in \mathcal{H}_{s}$. It follows from the definition of $\mathcal{H}_{s}$, that $(|A|+z I)^{\frac{s}{2}} u^{\prime} \in \mathcal{H}$, and

$$
\mathrm{u}(v)=\left(u^{\prime}, v\right)_{s}=\left((|A|+z I)^{\frac{s}{2}} u^{\prime},(|A|+z I)^{\frac{s}{2}} v\right)_{0},
$$

for $\operatorname{Re}(z)>0$. Therefore, we have a map

$$
\begin{aligned}
& (|\mathcal{A}|+z I)^{-\frac{s}{2}}: \mathcal{H}_{-s} \rightarrow \mathcal{H}, \\
& (|\mathcal{A}|+z I)^{-\frac{s}{2}}: \mathrm{u} \mapsto(|A|+z I)^{\frac{s}{2}} u^{\prime} .
\end{aligned}
$$

It is easy to see that the mapping $(|\mathcal{A}|+z I)^{-\frac{s}{2}}$ and $(|A|+z I)^{\frac{s}{2}}$ are inverse to each other. If we define the scalar product

$$
(\mathrm{u}, \mathrm{v})_{-s}=\left((|\mathcal{A}|+z I)^{-\frac{s}{2}} \mathrm{u},(|\mathcal{A}|+z I)^{-\frac{s}{2}} \mathrm{v}\right)_{0}
$$

both maps preserve this scalar product. We have proved that $(|\mathcal{A}|+z I)^{s}$ is an isometry of $\mathcal{H}_{t+2 s}$ onto $\mathcal{H}_{t}$, for all real $s$ and $t$, and all $z$ with $\operatorname{Re}(z)>0$, and $|\mathcal{A}|=|A|$ when both $s, t \geq 0$. Beside these isometries, we have the obvious inclusion $\mathcal{H}_{s} \leq \mathcal{H}_{t}$, for all $s \geq t$. We also note that, using the scalar product in $\mathcal{H}_{-s}$, the action of the functional $\mathrm{u} \in \mathcal{H}_{-s}$ is

$$
\mathbf{u}(v)=\left(\mathbf{u},(|A|+z I)^{s} v\right)_{-s}
$$

for all $v \in \mathcal{H}_{s}, \operatorname{Re}(z)>0$.

Lemma II.1 Let $A$ be a self adjoint operator in the Hilbert space $\mathcal{H}$, and $\mathrm{e} \in \mathcal{H}_{-2}-\mathcal{H}$. Then, the restriction $\dot{A}$ of $A$ defined by the domain

$$
\operatorname{dom}(\dot{A})=\{v \in \mathcal{H} \mid A v \in \mathcal{H}, \mathrm{e}(v)=0\}
$$

is symmetric, and has deficiency indices $(1,1)$. The solutions of the equation

$$
\left(\dot{A}^{\dagger} \mp i I\right) v=0,
$$

are all given by

$$
u_{ \pm}=c(|\mathcal{A}| \mp i I)^{-1} \mathrm{e}
$$

with $c \in \mathbb{C}$. 
Pooof. Note that the domain is well defined. For $A v \in \mathcal{H}$ if and only if $v \in \operatorname{dom}(A)$. First, we prove that $\dot{A}$ is symmetric. We show that $\operatorname{dom}(\dot{A})^{\perp}=\{0\}$, where the orthogonal complement is in $\mathcal{H}$. In fact, if this is the case, then

$$
\left(\operatorname{dom}(\dot{A})^{\perp}\right)^{\perp}=\mathcal{H}
$$

and $\left(\operatorname{dom}(\dot{A})^{\perp}\right)^{\perp}=\overline{\operatorname{dom}(\dot{A})}$, since $\operatorname{dom}(\dot{A})$ is a subspace, and the thesis follows. By definition

$$
\operatorname{dom}(\dot{A})^{\perp}=\left\{v \in \mathcal{H} \mid(v, w)_{0}=0, \forall w \in \operatorname{dom}(\dot{A})\right\} .
$$

We show that a vector $v$ satisfies the equation

$$
(v, w)_{0}=0,
$$

for all $w \in \operatorname{dom}(\dot{A})$, if and only if $v$ is a multiple of e under the inclusion of $\mathcal{H}_{\text {in }} \mathcal{H}_{-2}$ (one implication is obvious, since $w \in \operatorname{dom}(\dot{A}))$. This implies that the unique solution $v$ in $\mathcal{H}$ of the above equation is $v=0$. We have the following facts:

(a) if $w \in \operatorname{dom}(\dot{A}) \leq \mathcal{H}_{2}$, then

$$
(|A|+I) w=(|\mathcal{A}|+I)^{-1}(|\mathcal{A}|+I)^{2} w \in \mathcal{H}
$$

and hence $(|\mathcal{A}|+I)^{2} w \in \mathcal{H}_{-2} ;$

(b) by definition, $w \in \operatorname{dom}(\dot{A})$ if and only if

$$
0=\mathrm{e}(w)=\left(\mathrm{e},(|\mathcal{A}|+I)^{2} w\right)_{-2}
$$

(c) $\operatorname{dom}(\dot{A}) \leq \mathcal{H}_{2}$, and hence it is isometric to a subspace $D$ of $\mathcal{H}_{-2}, D=(|\mathcal{A}|+I)^{2} \operatorname{dom}(\dot{A})$;

(d) each $v \in \mathcal{H}$ defines a functional $\vee$ on $\mathcal{H}_{2}$ by $\vee(w)=(v, w)_{0}$; thus, we rewrite equation (8) as

$$
0=(v, w)_{0}=\mathrm{v}(w)=((|\mathcal{A}|+I) \mathrm{v},(|\mathcal{A}|+I) w)_{-2}=\left(\mathrm{v},(|\mathcal{A}|+I)^{2} w\right)_{-2},
$$

for all $w \in \operatorname{dom}(\dot{A})$, with $v=\mathrm{v} \in \mathcal{H} \leq \mathcal{H}_{-2}$.

By point (c), equation (9) means that $D=L(\mathrm{e})^{\perp_{-2}}$, where $\perp_{-2}$ means that the orthogonal complement is in $\mathcal{H}_{-2}$. By point (d), $v$ satisfies equation (8) if and only if $\mathrm{v} \in D^{\perp_{-2}}$. Since $\mathcal{H}_{-2}=L(\mathrm{e}) \oplus L(\mathrm{e})^{\perp_{-2}}$, it follows that $D^{\perp_{-2}}=\left(L(\mathrm{e})^{\perp_{-2}}\right)^{\perp_{-2}}=L(\mathrm{e})$, and hence $\vee$ satisfies equation (8) if and only if $\vee \in L(\mathrm{e})$, as required.

Next, we prove that the vectors $u_{ \pm}$are the unique solutions of the deficiency equation (7). Note that since e $\in \mathcal{H}_{-2}$, it follows that $u_{ \pm} \in \mathcal{H}$, and $\dot{A} u_{ \pm} \notin \mathcal{H}$. We show that $u_{ \pm} \in D(\dot{A} \dagger)$. By definition

$$
\operatorname{dom}\left(\dot{A}^{\dagger}\right)=\left\{v \in \mathcal{H} \mid \exists u \in \mathcal{H},(\dot{A} w, v)_{0}=(w, u)_{0}, \forall w \in \operatorname{dom}(\dot{A})\right\}
$$

If we take $u= \pm i u_{ \pm}$, then

$$
\begin{aligned}
\left(\dot{A} w, u_{ \pm}\right)_{0}-\left(w, \pm i u_{ \pm}\right)_{0} & =\left(\dot{A} w, u_{ \pm}\right)_{0}-\left(\mp i w, u_{ \pm}\right)_{0}=\left((\dot{A} \pm i I) w, u_{ \pm}\right)_{0} \\
& =\left((A \pm i I) w, u_{ \pm}\right)_{0}=\left(w,(A \mp i I) u_{ \pm}\right)_{0}=\overline{\mathrm{e}(w)}=0
\end{aligned}
$$

since $A=\dot{A}$ on $\operatorname{dom}(\dot{A})$, and $A$ is self adjoint, and hence $u_{ \pm}$belong to $\operatorname{dom}\left(\dot{A}^{\dagger}\right)$. This also means that $\dot{A}^{\dagger} u_{ \pm}=i u_{ \pm}$, and therefore the $u_{ \pm}$are solutions of equation (7). It remains to show that these are the unique solutions. For, note that the solutions of equation (7) are elements of the space

$$
\operatorname{ker}\left(i I-\dot{A}^{\dagger}\right)=(\operatorname{ran}(-i I-\dot{A}))^{\perp},
$$

and $u \in(\operatorname{ran}(-i I-\dot{A}))^{\perp}$ if and only if

$$
(u,(-i I-\dot{A}) w)_{0}=0,
$$

for all $w \in \operatorname{dom}(\dot{A})$. By point (c) above, if $w \in \operatorname{dom}(\dot{A})$, then $(|\mathcal{A}|+I)^{2} w \in \mathcal{H}_{-2}$, so equation (11) means that

$$
\begin{aligned}
0 & =(u,(-i I-\dot{A}) w)_{0}=(u,(-i I-A) w)_{0}=\left(u,(-i I-A)(|\mathcal{A}|+I)^{-2}(|\mathcal{A}|+I)^{2} w\right)_{0} \\
& =\left((i I-\mathcal{A}) \mathbf{u},(|\mathcal{A}|+I)^{2} w\right)_{-2},
\end{aligned}
$$

since $A$ is self adjoint. This implies that $(i I-\mathcal{A}) \mathbf{u} \in D^{\perp_{-}}$(where the space $D$ was defined in point (c) above). Since $D^{\perp-2}=L(\mathrm{e})$, this completes the proof.

Using the standard von Neumann theory of self adjoint extensions, we characterize the adjoint and the self adjoint extensions of $\dot{A}$ as follows. 
Lemma II.2 The adjoint operator $\dot{A}^{\dagger}$ is

$$
\begin{aligned}
\operatorname{dom}\left(\dot{A}^{\dagger}\right) & =\left\{w \oplus c_{+} u_{+} \oplus c_{-} u_{-}, w \in \operatorname{dom}(\dot{A}), c_{ \pm} \in \mathbb{C}\right\}, \\
\dot{A}^{\dagger}\left(w+u_{+}+u_{-}\right) & =\dot{A} w+i u_{+}-i u_{-} .
\end{aligned}
$$

Lemma II.3 All the self adjoint extensions $A_{\theta}, 0 \leq \theta<2 \pi$, of the operator $\dot{A}$ are

$$
\begin{aligned}
\operatorname{dom}\left(A_{\theta}\right) & =\left\{w \oplus c_{+}\left(u_{+} \oplus \mathrm{e}^{i \theta} u_{-}\right), w \in \operatorname{dom}(\dot{A}), c_{+} \in \mathbb{C}\right\}, \\
A_{\theta}\left(w+u_{+}+u_{-}\right) & =\dot{A}^{\dagger}\left(w+u_{+}+u_{-}\right)=\dot{A} w+i u_{+}-i u_{-} .
\end{aligned}
$$

For proofs of these Lemmas see for example [3], [4]. An equivalent description of the self adjoint extensions can be given by boundary conditions on the domain of the adjoint operator as

$$
\operatorname{dom}\left(A_{\theta}\right)=\left\{u \in \operatorname{dom}\left(\dot{A}^{\dagger}\right) \mid\left(u_{-}, u\right)_{0}=\mathrm{e}^{i \theta}\left(u_{+}, u\right)_{0}\right\} .
$$

Remark II.4 Note that the case $\theta=\pi$ gives the maximal operator, namely $A_{\theta} \subseteq A_{\pi}$, for all $\theta$. For $v \in \operatorname{dom}\left(Q_{\pi}\right)$ if and only if $v=w+c_{+}\left(u_{+}-u_{-}\right)$, with $w \in \operatorname{dom}(\dot{A})$, and $c_{+} \in \mathbb{C}$. But it is easy to see that the function $u=u_{+}-u_{-}=2 i\left(\mathcal{A}^{2}+I\right)^{-1} \mathrm{e}$ is such that $u \in L^{2}\left(\Omega^{n}\right)$ and $\dot{A} u \in L^{2}\left(\Omega^{n}\right)$. This means that $u \in \operatorname{dom}(A)$, and the statement follows.

Next we characterize the resolvent of the self adjoint extensions $A_{\theta}$ of $\dot{A}$. This should be compared with Theorem 1.2 .1 of $[\underline{3}]$.

Lemma II.5 Let $A_{\theta}$ be one of the self adjoint extensions of the operator $\dot{A}$ described in Lemma II.3. Let $\lambda \in$ $\rho\left(A_{\theta}\right) \cap \rho\left(A_{\pi}\right)$, then the resolvent of $A_{\theta}$ of $\dot{A}$ is

$$
R\left(\lambda, A_{\theta}\right) v=R\left(\lambda, A_{\pi}\right) v+c_{\theta}(\lambda)\left(u_{\bar{\lambda}}, v\right)_{0} u_{\lambda},
$$

where $c_{\theta}(\lambda)$ is some function of $\lambda$, and

$$
u_{\lambda}=(|\mathcal{A}|-\lambda I)^{-1} \mathrm{e} .
$$

Moreover, the difference of the resolvents $R\left(\lambda, A_{\theta}\right)-R\left(\lambda, A_{\pi}\right)$ is of trace class, and

$$
\operatorname{Tr}\left(R\left(\lambda, A_{\theta}\right)-R\left(\lambda, A_{\pi}\right)\right)=c_{\theta}(\bar{\lambda})\left(u_{\bar{\lambda}}, u_{\lambda}\right)_{0} .
$$

Pooof. Let $\lambda \in \rho\left(A_{\theta}\right) \cap \rho\left(A_{\pi}\right)$. Consider

$$
\left(R\left(\lambda, A_{\theta}\right)-R\left(\lambda, A_{\pi}\right)\right) v=\left(\left(\lambda I-A_{\theta}\right)^{-1}-\left(\lambda I-A_{\pi}\right)^{-1}\right) v .
$$

Since for all $u \in \operatorname{ran}(\lambda I-\dot{A})$, we have that $\left(\lambda I-A_{\theta}\right)^{-1} u=(\lambda I-\dot{A})^{-1} u$, it follows that

$$
\left(R\left(\lambda, A_{\theta}\right)-R\left(\lambda, A_{\pi}\right)\right) v=\left(\left(\lambda I-A_{\theta}\right)^{-1}-\left(\lambda I-A_{\pi}\right)^{-1}\right) P_{(\operatorname{ran}(\lambda I-\dot{A})) \perp} v,
$$

where $P_{(\operatorname{ran}(\lambda I-\dot{A}))^{\perp}}$ denotes the projection onto $(\operatorname{ran}(\lambda I-\dot{A}))^{\perp}$. But it is easy to see that the proof of Lemma ЏI.6 generalizes for any $\lambda \neq i$ in the resolvent set, thus $(\operatorname{ran}(\lambda I-\dot{A}))^{\perp}=<u_{\bar{\lambda}}>$, with

$$
u_{\lambda}=(|\mathcal{A}|-\lambda I)^{-1} \mathrm{e} .
$$

It follows that,

$$
\left(R\left(\lambda, A_{\theta}\right)-R\left(\lambda, A_{\pi}\right)\right) v=\left(\left(\lambda I-A_{\theta}\right)^{-1}-\left(\lambda I-A_{\pi}\right)^{-1}\right)\left(u_{\bar{\lambda}}, v\right)_{0} u_{\bar{\lambda}} .
$$

Now, the vector $y=\left(\left(\lambda I-A_{\theta}\right)^{-1}-\left(\lambda I-A_{\pi}\right)^{-1}\right) u_{\bar{\lambda}}$ itself belongs to $(\operatorname{ran}(\bar{\lambda} I-\dot{A}))^{\perp}$. For, since $\dot{A}^{\dagger}$ is an extension of $A_{\theta}$ for all $\theta$,

$$
\left(\lambda I-\dot{A}^{\dagger}\right) y=\left(\lambda I-\dot{A}^{\dagger}\right)\left(\left(\lambda I-A_{\theta}\right)^{-1}-\left(\lambda I-A_{\pi}\right)^{-1}\right) u_{\bar{\lambda}}=0,
$$

implies that $y \in \operatorname{ker}\left(\lambda I-\dot{A}^{\dagger}\right)$. Therefore, we have proved that for all $v \in \operatorname{dom}\left(\lambda I-A_{\theta}\right)^{-1}$,

$$
\left(R\left(\lambda, A_{\theta}\right)-R\left(\lambda, A_{\pi}\right)\right) v=\left(u_{\bar{\lambda}}, v\right)_{0} c_{\theta}(\lambda) u_{\lambda} .
$$

This means that

$$
\operatorname{Tr}\left(R\left(\lambda, A_{\theta}\right)-R\left(\lambda, A_{\pi}\right)\right)=c_{\theta}(\bar{\lambda})\left(u_{\bar{\lambda}}, u_{\lambda}\right)_{0}<\infty,
$$

by the definition of $u_{\lambda}$, since $\mathrm{e} \in \mathcal{H}_{-2}$. 


\section{B. Multiplication operators}

We pass now to consider a more concrete situation, namely multiplication operators. These operators provide the most natural setting where the results given in the previous section for abstract operators apply. Dually, all the results of the present section can be proved independently from the theory developed in Section II but working directly in the concrete Sobolev spaces described below. We will not give complete proofs in this concrete setting, since they are precisely the same as the one provided in the abstract presentation of Section IA The main advantage working in this concrete setting, is that all the spaces $\mathcal{H}_{s}$ of Section $\amalg \mathrm{A}$ are subspaces of some large function space, and therefore all the functionals can be identified with some concrete functions in these spaces. First, some preliminaries and notations. The measure appearing in all integrals is going to be Lebesgue's measure. Correspondingly, measurable sets and functions are understood in the sense of Lebesgue's integration theory. Let $\Omega^{n}$ be some unbounded measurable subset of $\mathbb{R}^{n}$. Let $s$ be real, $q: \Omega^{n} \rightarrow \mathbb{C}$ be a measurable function, and

$$
m_{s}(x)=(1+|q(x)|)^{\frac{s}{2}} .
$$

We define the spaces

$$
L^{2, s}\left(\Omega^{n}\right)=\left\{f \in \operatorname{map}\left(\Omega^{n}, \mathbb{C}\right) \mid f m_{s} \in L^{2}\left(\Omega^{n}\right)\right\} .
$$

Note that, $L^{2}\left(\Omega^{n}\right)=L^{2,0}\left(\Omega^{n}\right)$, and $L^{2, s}\left(\Omega^{n}\right) \subset L^{2, s^{\prime}}\left(\Omega^{n}\right)$ if $s>s^{\prime}$. In $L^{2, s}\left(\Omega^{n}\right)$ we have the scalar product

$$
(f, g)_{L^{2, s}\left(\Omega^{n}\right)}=\int_{\Omega^{n}} \bar{f}(x) g(x)\left(m_{s}(x)\right)^{2} d^{n} x,
$$

and with this scalar product, the spaces $L^{2, s}\left(\Omega^{n}\right)$ are complete separable Hilbert spaces and are the Fourier images of the Sobolev spaces $W^{2, s}\left(\Omega^{n}\right)$. We will use the notation $B_{N}^{n}$ for the intersection of $\Omega^{n}$ and the closed disc $D_{N}^{n}$ of radius $N$ centered in the origin of $\mathbb{R}^{n}$.

The maximal multiplication operator $Q$ associated to $q$ is the operator in $L^{2}\left(\Omega^{n}\right)$ defined by

$$
\begin{aligned}
\operatorname{dom}(Q) & =\left\{f \in L^{2}\left(\Omega^{n}\right) \mid q f \in L^{2}\left(\Omega^{n}\right)\right\}, \\
Q f & =q f .
\end{aligned}
$$

The operator $Q$ is a (closed) self adjoint operator with core $C_{0}^{\infty}\left(\Omega^{n}\right)$. If $q(x) \neq 0$ a.e. in $\Omega^{n}$, then $Q$ is injective. If $q(x) \geq c$, for some $c$, a.e. in $\Omega^{n}$, then $\operatorname{ran}(Q)=L^{2}\left(\Omega^{n}\right)$, so $Q: \operatorname{dom}(Q) \rightarrow L^{2}\left(\Omega^{n}\right)$ is bijective.

\section{Non maximal multiplication operators and their extensions}

Let $e: \Omega^{n} \rightarrow \mathbb{C}$ be a fixed measurable function. Assume the functions $q$ and $e$ decrease faster than some power, namely there exist constants $\mu, \beta, c$, and $c^{\prime}$ such that

$$
\begin{aligned}
& \left|x^{-\mu} q(x)\right| \leq c, \\
& \left|x^{-\beta} e(x)\right| \leq c^{\prime} .
\end{aligned}
$$

Consider in $L^{2}\left(\Omega^{n}\right)$ the operator

$$
\begin{aligned}
\operatorname{dom}(\dot{Q}) & =\left\{f \in L^{2}\left(\Omega^{n}\right) \mid q f \in L^{2}\left(\Omega^{n}\right), \int_{\Omega^{n}} \bar{e}(x) f(x) d^{n} x=0\right\}, \\
\dot{Q} f & =q f .
\end{aligned}
$$

It is clear that $\operatorname{dom}(\dot{Q})$ is a subspace of $L^{2}\left(\Omega^{n}\right)$, so the definition is well posed. Also, $\operatorname{dom}(\dot{Q}) \subset \operatorname{dom}(Q)$, so $\dot{Q}$ is a multiplication operator, but in general it is not maximal.

Lemma II.6 If $\mu-\beta>\frac{n}{2}$, then $\dot{Q}$ is symmetric.

Pooof. This follows from the first statement of Lemma II.1 provided that $e \in \mathcal{H}_{-2}$. In the present case, $|A|=|Q|$, and therefore $\mathcal{H}_{-2}=L^{2,-2}\left(\Omega^{n}\right)$. Thus, $e \in \mathcal{H}_{-2}$ if

$$
\int_{\Omega^{n}}|e(x)|^{2}(1+|q(x)|)^{-2} d^{n} x<\infty .
$$

We only need to check the convergence for large $r=|x|$. For large $r$ the integral behaves like $r^{2 \beta-2 \mu+n-1}$, and therefore it is convergent if $2 \beta-2 \mu+n<0$. 
Lemma II.7 Assume $q$ is a real function, $\mu-\beta>\frac{n}{2}$, and $\beta \geq-\frac{n}{2}$. Then the operator $\dot{Q}$ has deficiency indices $(1,1)$, and the solutions of the equation

$$
\left(\dot{Q}^{\dagger} \pm i I\right) u=0
$$

are all given by the functions

$$
\psi_{ \pm}(x)=c \frac{e(x)}{q(x) \mp i}
$$

where $c \in \mathbb{C}, x \in \Omega^{n}$.

Pooof. This follows from the second statement of Lemma II.1, provided that $e \in \mathcal{H}_{-2}$ and that $q \psi_{ \pm} \notin L^{2}\left(\Omega^{n}\right)$. The first requirements implies $\mu-\beta>\frac{n}{2}$ as in the proof of the previous lemma. For the second one, consider the integral

$$
\int_{\Omega^{n}}\left|q(x) \psi_{+}(x)\right|^{2} d^{n} x=\int_{\Omega^{n}} \frac{|q(x) e(x)|^{2}}{|q(x)+1|^{2}} d^{n} x .
$$

This integral behaves for large $r=|x|$ as $r^{2 \beta+n}$, and therefore does not converge if $\beta \geq \frac{n}{2}$.

Remark II.8 Note that the conditions $\mu-\beta>\frac{n}{2}$ and $\beta \geq-\frac{n}{2}$ imply that $\mu>0$. For $-\beta \leq \frac{n}{2}$, and hence $\frac{n}{2}<\mu-\beta \leq \mu+\frac{n}{2}$.

We are now in the position of using the results in Lemmas $₫ .2$ and $\llbracket .3$ to characterize the adjoint of $\dot{Q}$ and to parameterize all the self adjoint extensions, using the parameter $\theta$. With this parameterization, the maximal multiplication operator $Q$ corresponds to the self adjoint extension defined by $\theta=\pi$ (see remark II.4). Using Lemma II.5 we also have a general formula for the resolvent and we know that the difference between the resolvents of a self adjoint extension and of the maximal operator is of trace class.

We proceed by studying the particular case where $q(x)=|x|^{2}$ and $e$ is a bounded function. In this case, we give a more useful characterization of the self adjoint extensions of $\dot{Q}$ by some explicit integral boundary conditions. We will parameterize the self adjoint extensions by a real non negative parameter $\alpha$. By the assumptions on $q$ and $e$, we have $\mu=2$ and $\beta=0$, and the conditions in Lemmas II.6 and II.7 are satisfied if and only if $n=1,2$ or 3. Therefore, we proceed assuming $n$ to be in this range. In this case, if

$$
\psi_{\lambda}(x)=\frac{e(x)}{|x|^{2}-\lambda},
$$

and assuming $\operatorname{Im} \lambda \neq 0$, we have the following expansion for large $N$

$$
\int_{B_{N}^{n}} \bar{e}(x) \psi_{\lambda}(x) d^{n} x=\int_{B_{N}^{n}} \frac{|e(x)|^{2}}{|x|^{2}-\lambda} d^{n} x=d_{n}(N)+g_{n}(\lambda)+o\left(N^{\left[\frac{n}{2}\right]-1}\right), \quad n=1,2,3 .
$$

Note that $d_{n}(N)$ does not depend on $\lambda$. The functions $d_{n}(N)$ and $g_{n}(\lambda)$ depend on the explicit form of $e(x)$. The values for the relevant choices of $e(x)$ are given in Lemma V.1 in the appendix. In particular, it is always true that

$$
d_{1}(N)=O\left(\frac{1}{N}\right), \quad d_{2}(N)=O(\log N), \quad d_{3}(N)=O(N) .
$$

From equation (12) it follows that

$$
\begin{aligned}
\int_{B_{N}^{n}} \bar{e}(x)\left(\psi_{+}(x)+\mathrm{e}^{i \theta} \psi_{-}(x)\right) d^{n} x & =\left(1+\mathrm{e}^{i \theta}\right) d_{n}(N)+g_{n}(i)+\mathrm{e}^{i \theta} g_{n}(-i)+o\left(N^{\left[\frac{n}{2}\right]-1}\right) \\
& =i\left(1-\mathrm{e}^{i \theta}\right)\left(\alpha d_{n}(N)+\alpha b_{n}+a_{n}\right)+o\left(N^{\left[\frac{n}{2}\right]-1}\right),
\end{aligned}
$$

where $\alpha, a_{n}, b_{n}$ are real numbers given by

$$
\begin{aligned}
\alpha & =\operatorname{ctg} \frac{\theta}{2} \\
a_{n} & =\left\|\psi_{+}\right\|^{2}=\left\|\psi_{-}\right\|^{2}=\int_{\Omega^{n}} \frac{|e(x)|^{2}}{1+x^{4}} d^{n} x=\frac{g_{n}(i)-g_{n}(-i)}{2 i}, \\
b_{n} & =\frac{g_{n}(i)+g_{n}(-i)}{2} .
\end{aligned}
$$


Therefore, $\alpha$ is the real number which specifies the chosen self adjoint extension.

This suggests the following description of the boundary conditions of the self adjoint extensions of $\dot{Q}$ in all the cases $n=1,2,3$.

Proposition II.9 All the self adjoint extensions of the operator $\dot{Q}$ are given by the following integral boundary condition

$$
\begin{aligned}
\operatorname{dom}\left(Q_{\alpha}\right)= & \left\{f \in L^{2}\left(\Omega^{n}\right) \mid q f-\alpha c e \in L^{2}\left(\Omega^{n}\right),\right. \\
& \left.\int_{B_{N}^{n}} \bar{e}(x) f(x) d^{n} x=c\left(\alpha d_{n}(N)+\alpha b_{n}+a_{n}\right)+o(1) \text {, for large } N\right\},
\end{aligned}
$$

$c$ being an arbitrary complex constant, $d_{n}(N)$ the functions given in equation (12), and $\alpha$ a real number. The operator $Q_{\alpha}$ acts on $f \in \operatorname{dom}\left(Q_{\alpha}\right)$ as follows

$$
\begin{aligned}
\left(Q_{\alpha} f\right)(x) & =|x|^{2} f(x)-\alpha c e(x) \\
& =|x|^{2} f(x)-\frac{2 \alpha}{\alpha-i}\left(\psi_{+}, f\right) e(x) \\
& =|x|^{2} f(x)-\lim _{N \rightarrow \infty} \frac{\alpha e(x)}{\alpha d_{n}(N)+\alpha b_{n}+a_{n}} \int_{B_{N}^{n}} \bar{e}(x) f(x) d^{n} x .
\end{aligned}
$$

Remark II.10 In dimensions two and three, $d_{n}(N)$ are divergent quantities and so the constants $a_{n}, b_{n}$ can be dropped in the latter equation, while in dimension $n=1$ one can drop $d_{1}(N)$ and take the integral on the whole space.

Pooof. Let $f \in \operatorname{dom}\left(Q_{\alpha}\right)$, that is $f=h+c_{+}\left(\psi_{+}+\mathrm{e}^{i \theta} \psi_{-}\right)$with $h \in \operatorname{dom}(\dot{Q})$. Then,

$$
\begin{aligned}
\int_{B_{N}^{n}} \bar{e}(x) f(x) d^{n} x & =\int_{B_{N}^{n}} \bar{e}(x) h(x) d^{n} x+c_{+} \int_{B_{N}^{n}} \bar{e}(x)\left(\psi_{+}(x) d^{n} x+\mathrm{e}^{i \theta} \psi_{-}(x)\right) d^{n} x \\
& =\int_{B_{N}^{n}} \bar{e}(x) h(x) d^{n} x+c\left(\alpha d_{n}(N)+\alpha b_{n}+a_{n}\right)+o\left(N^{\left[\frac{n}{2}\right]-1}\right),
\end{aligned}
$$

with $c=i\left(1-\mathrm{e}^{i \theta}\right) c_{+}$. Now we have to show that for large $N$, the integral in the latter equation always gives contributions which are negligible with respect $\alpha\left(d_{n}(N)+b_{n}\right)+a_{n}$. This is however a direct consequence of the fact that $h \in \operatorname{dom}(\dot{Q})$. For by hypothesis we have

$$
\int_{\Omega^{n}} \bar{e}(x) h(x) d^{n} x=0
$$

since $\Omega^{n}=\bigcup_{N} B_{N}^{n}$, this implies that

$$
\lim _{N \rightarrow \infty} \int_{B_{N}^{n}} \bar{e}(x) h(x) d^{n} x=0
$$

namely that

$$
\int_{B_{N}^{n}} \bar{e}(x) h(x) d^{n} x=o(1)
$$

for large $N$.

The formula for the action of $Q_{\alpha}$ easily follows.

Next, we give the resolvent of each extension.

Proposition II.11 Let $Q_{\alpha}$ be one of the self adjoint extensions of the non maximal multiplication operator $\dot{Q}$ described in Proposition II.9. Then, for all $\lambda \in \rho\left(Q_{\alpha}\right) \cap \rho\left(Q_{0}\right), Q_{\alpha}$ has the resolvent

$$
\begin{aligned}
R\left(\lambda, Q_{\alpha}\right) \phi & =R\left(\lambda, Q_{0}\right) \phi+\frac{1}{\frac{a_{n}}{\alpha}+b_{n}-g_{n}(\lambda)} \int_{\Omega^{n}} \bar{e}(x)\left(R\left(\lambda, Q_{0}\right) \phi\right)(x) d^{n} x \psi_{\lambda}, \\
\psi_{\lambda}(x) & =\frac{e(x)}{|x|^{2}-\lambda},
\end{aligned}
$$


where the $g_{n}(\lambda)$ are the functions appearing in the asymptotic expansion of the integral boundary condition defining $Q_{\alpha}$ applied to the function $\psi_{\lambda}$, as given in equation (12), and $Q_{0}$ is the maximal multiplication operator $Q$. Moreover, the difference of the resolvents $R\left(\lambda, Q_{\alpha}\right)-R\left(\lambda, Q_{0}\right)$ is of trace class.

Pooof. By Lemma II.5.

$$
R\left(\lambda, Q_{\alpha}\right) \phi=R\left(\lambda, Q_{0}\right) \phi+c_{\alpha}(\lambda, \phi) \psi_{\lambda}
$$

In order to find the value of $c_{\alpha}(\lambda, \phi)$, first we note that $f=R\left(\lambda, Q_{\alpha}\right) \phi \in \operatorname{dom}\left(Q_{\alpha}\right)$, therefore it must satisfies the conditions given in Proposition II.9, namely

$$
\int_{B_{N}^{n}} \bar{e}(x) f(x) d^{n} x=\int_{B_{N}^{n}} \bar{e}(x)\left(R\left(\lambda, Q_{\alpha}\right) \phi\right)(x) d^{n} x=c\left(a_{n}+\alpha b_{n}+\alpha d_{n}(N)\right)+o(1) .
$$

On the other hand, using equation (12) we explicitly have

$$
\begin{aligned}
\int_{B_{N}^{n}} \bar{e}(x)\left(R\left(\lambda, Q_{\alpha}\right) \phi\right)(x) d^{n} x= & \int_{B_{N}^{n}} \bar{e}(x)\left(R\left(\lambda, Q_{0}\right) \phi\right)(x) d^{n} x \\
& +c_{\alpha}(\lambda, \phi) \int_{B_{N}^{n}} \bar{e}(x) \psi_{\lambda}(x) d^{n} x \\
= & \int_{B_{N}^{n}} \bar{e}(x)\left(R\left(\lambda, Q_{0}\right) \phi\right)(x) d^{n} x \\
& +c_{\alpha}(\lambda, \phi)\left(d_{n}(N)+g_{n}(\lambda)\right)+o\left(N^{\left[\frac{n}{2}\right]-1}\right) .
\end{aligned}
$$

Now, since $R\left(\lambda, Q_{0}\right) \phi \in \operatorname{dom}\left(Q_{0}\right)$, by Proposition $\amalg .9$

$$
\int_{B_{N}^{n}} \bar{e}(x)\left(R\left(\lambda, Q_{0}\right) \phi\right)(x) d^{n} x=c a_{n}+o(1)
$$

for large $N$. Note that the constant $a_{n}$ does not depend on the extension by definition.

This means that we are able to make the comparison between the two equations (13) and (14). We have

$$
\int_{\Omega^{n}} \bar{e}(x)\left(R\left(\lambda, Q_{0}\right) \phi\right)(x) d^{n} x+c_{\alpha}(\lambda, \phi)\left(d_{n}(N)+g_{n}(\lambda)\right)-c\left(a_{n}+\alpha b_{n}+\alpha d_{n}(N)\right)=o(1),
$$

and this implies that $c_{\alpha}(\lambda, \phi)=\alpha c$ and

$$
c_{\alpha}(\lambda, \phi)=\frac{\alpha}{a_{n}+\alpha b_{n}-\alpha g_{n}(\lambda)} \int_{\Omega^{n}} \bar{e}(x)\left(R\left(\lambda, Q_{0}\right) \phi\right)(x) d^{n} x=\frac{1}{\frac{a_{n}}{\alpha}+b_{n}-g_{n}(\lambda)} \int_{\Omega^{n}} \frac{\bar{e}(x) \phi(x)}{\lambda-|x|^{2}} d^{n} x
$$

Note that in the case $n=1$, we are comparing constants, since $d_{1}(N)$ is small in this case (see Remark II.10).

In order to give the kernel of the resolvent, we need a suitable delta function in the space $\Omega^{n}$. This will be denoted by $\delta_{\Omega^{n}}$, and is defined by the property

$$
\int_{\Omega^{n}} \delta_{\Omega^{n}}(x-a) f(x) d^{n} x=f(a)
$$

in the appropriate space of test functions over $\mathbb{R}^{n}$. Explicit formulas will be given in the concrete examples studied below.

Corollary II.12 The operator $R\left(\lambda, Q_{\alpha}\right)$ of Proposition II.11 is an integral operator with kernel

$$
\operatorname{ker}\left(x, y ; R\left(\lambda, Q_{\alpha}\right)\right)=\frac{\delta_{\Omega^{n}}(x-y)}{\lambda-|x|^{2}}-\frac{1}{\frac{a_{n}}{\alpha}+b_{n}-g_{n}(\lambda)} \frac{e(x) \bar{e}(y)}{\left(\lambda-|x|^{2}\right)\left(\lambda-|y|^{2}\right)}
$$

Pooof. Since

$$
\left(R\left(\lambda, Q_{0}\right) \phi\right)(x)=\frac{\phi(x)}{\lambda-|x|^{2}}
$$


we have that the operator

$$
\begin{aligned}
\left(A_{\lambda} \phi\right)(x) & =\psi_{\lambda}(x) \int_{\Omega^{n}} \bar{e}(y)\left(R\left(\lambda, Q_{0}\right) \phi\right)(y) d^{n} y \\
& =\psi_{\lambda}(x) \int_{\Omega^{n}} \bar{e}(y) \frac{\phi(y)}{\lambda-|y|^{2}} d^{n} y=\int_{\Omega^{n}} \frac{e(x) \bar{e}(y)}{\left(|x|^{2}-\lambda\right)\left(\lambda-|y|^{2}\right)} \phi(y) d^{n} y,
\end{aligned}
$$

is an integral operator with kernel

$$
\operatorname{ker}(x, y ; A(\lambda))=-\frac{e(x)}{\lambda-|x|^{2}} \frac{\bar{e}(y)}{\lambda-|y|^{2}} .
$$

Corollary II.13 The difference of the resolvents $R\left(\lambda, Q_{\alpha}\right)-R\left(\lambda, Q_{0}\right)$ of Proposition II.11 is a trace class operator with trace

$$
\operatorname{Tr}\left(R\left(\lambda, Q_{\alpha}\right)-R\left(\lambda, Q_{0}\right)\right)=-\frac{1}{\frac{a_{n}}{\alpha}+b_{n}-g_{n}(\lambda)} \int_{\Omega^{n}} \frac{|e(x)|^{2}}{\left(|x|^{2}-\lambda\right)^{2}} d^{n} x
$$

Remark II.14 Assuming $\operatorname{Im} \lambda \neq 0$, we have the bound

$$
\left|\operatorname{Tr}\left(R\left(\lambda, Q_{\alpha}\right)-R\left(\lambda, Q_{0}\right)\right)\right| \leq K \frac{1}{\left|\frac{a_{n}}{\alpha}+b_{n}-g_{n}(\lambda)\right|}\left|\lambda^{\frac{n}{2}-2}\right|,
$$

with some positive constant K. For by Corollary II.12

$$
\operatorname{ker}\left(x, y ; R\left(\lambda, Q_{\alpha}\right)-R\left(\lambda, Q_{0}\right)\right)=-\frac{1}{\frac{a_{n}}{\alpha}+b_{n}-g_{n}(\lambda)} \frac{e(x) \bar{e}(y)}{\left(\lambda-|x|^{2}\right)\left(\lambda-|y|^{2}\right)} .
$$

Since $\operatorname{Im} \lambda \neq 0$ we can integrate obtaining

$$
\begin{aligned}
\left|\operatorname{Tr}\left(R\left(\lambda, Q_{\alpha}\right)-R\left(\lambda, Q_{0}\right)\right)\right| & =\frac{1}{\left|\frac{a_{n}}{\alpha}+b_{n}-g_{n}(\lambda)\right|} \int_{\Omega^{n}} \frac{|e(x)|^{2}}{\left(|x|^{2}-\lambda\right)^{2}} d^{n} x \\
& \leq \frac{1}{\left|\frac{a_{n}}{\alpha}+b_{n}-g_{n}(\lambda)\right|} \int_{\mathbb{R}^{n}} \frac{|e(x)|^{2}}{\left(|x|^{2}-\lambda\right)^{2}} d^{n} x \\
& \leq \frac{K}{\left|\frac{a_{n}}{\alpha}+b_{n}-g_{n}(\lambda)\right|}\left|\lambda^{\frac{n}{2}-2}\right| .
\end{aligned}
$$

In the latter line we have used Lemma $V .4$ in the appendix.

We conclude this section giving the continuum spectrum of the operators $Q_{\alpha}$. Possible isolated eigenvalues of finite multiplicity will be detected by an explicit study of the resolvent of particular examples in the following sections.

Lemma II.15 The continuum spectrum of the operator $Q_{\alpha}$ coincides with the non negative real axis, i.e. $\operatorname{Sp}_{c} Q_{\alpha}=$ $[0, \infty)$.

Pooof. We recall that $\mathrm{Sp}_{c} \subseteq \mathrm{Sp}_{e}$, for closed operators, and $\mathrm{Sp}_{e}=\overline{\mathrm{Sp}_{c}} \cup \overline{\mathrm{Sp}_{p}} \cup \mathrm{Sp}_{p}^{\infty}$, for self adjoint operators. Then, the thesis follows since all self adjoint extensions have the same essential spectrum, and the maximal operator $Q_{0}$ is known to have the pure continuous spectrum $\operatorname{Sp} Q_{0}=\operatorname{Sp}_{c} Q_{0}=[0, \infty)$.

\section{The basic example in the whole space}

Let $\Omega^{n}=\mathbb{R}^{n}, e(x)=\mathrm{e}^{i a x}$, where $a \in \mathbb{R}^{n}$, and $n=1,2,3$. We study in this section the self adjoint extensions of the (closure of the) operator $\dot{Q}$ in $L^{2}\left(\mathbb{R}^{n}\right)$ defined by

$$
\begin{aligned}
\operatorname{dom}(\dot{Q}) & =\left\{f \in L^{2}\left(\mathbb{R}^{n}\right) \mid q f \in L^{2}\left(\mathbb{R}^{n}\right), \int_{\mathbb{R}^{n}} \mathrm{e}^{-i a x} f(x) d^{n} x=0\right\}, \\
\dot{Q} f & =q f
\end{aligned}
$$


where $q(x)=|x|^{2}$. We have $|e|=1$, and $B_{N}^{n}=D_{N}^{n}$. In order to apply Proposition 【.9, we have to compute the functions $d_{n}(N)$ and $g_{n}(\lambda)$ appearing in equation (12). They are explicitly given in Lemma V.1 in the appendix.

In the following we shall assume $\operatorname{Im} \sqrt{\lambda}>0$, then, from Lemma $\mathrm{V} .1$ in the appendix, for $n=3,2,1$ we have respectively

$$
\begin{aligned}
& n=3, \quad\left\{\begin{array}{l}
\int_{D_{N}^{3}} \frac{1}{\left.x\right|^{2}-\lambda} d^{3} x=4 \pi N+2 \pi^{2} i \sqrt{\lambda} \operatorname{sgn}(\operatorname{Im} \sqrt{\lambda})+o(1), \\
d_{3}(N)=4 \pi N, \quad g_{3}(\lambda)=2 \pi^{2} i \sqrt{\lambda}, \quad a_{3}=\sqrt{2} \pi^{2}, \quad b_{3}=-\sqrt{2} \pi^{2},
\end{array}\right. \\
& n=2, \quad\left\{\begin{array}{l}
\int_{D_{N}^{2}} \frac{1}{|x|^{2}-\lambda} d^{2} x=2 \pi \log N-\pi \log (-\lambda)+o(1), \\
d_{2}(N)=2 \pi \log N, \quad g_{2}(\lambda)=-\pi \log (-\lambda), \quad a_{2}=\frac{\pi^{2}}{2}, \quad b_{2}=0,
\end{array}\right. \\
& n=1, \quad\left\{\begin{array}{l}
\int_{D_{N}^{1}} \frac{1}{|x|^{2}-\lambda} d x=\frac{i \pi}{\sqrt{\lambda} \operatorname{sgn}(\operatorname{Im} \sqrt{\lambda})}-\frac{2}{N}+o\left(\frac{1}{N}\right), \\
d_{1}(N)=-\frac{2}{N}, \quad g_{1}(\lambda)=\frac{i \pi}{\sqrt{\lambda}}, \quad a_{1}=\frac{\pi}{\sqrt{2}}, \quad b_{1}=\frac{\pi}{\sqrt{2}} .
\end{array}\right.
\end{aligned}
$$

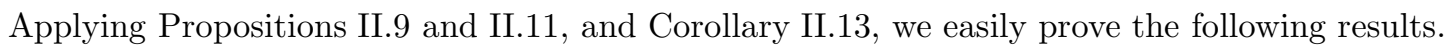

Lemma II.16 The self adjoint extensions of $\dot{Q}$ are:

$$
\begin{aligned}
& n=3, \quad\left\{\begin{aligned}
& \operatorname{dom}\left(Q_{\alpha}\right)=\left\{f \in L^{2}\left(\mathbb{R}^{3}\right) \mid Q_{\alpha} f \in L^{2}\left(\mathbb{R}^{3}\right),\right. \\
&\left.\int_{D_{N}^{3}} \mathrm{e}^{-i a x} f(x) d^{3} x=c\left(4 \pi N \alpha-\sqrt{2} \pi^{2} \alpha+\sqrt{2} \pi^{2}\right)+o(1), c \in \mathbb{C}\right\}, \\
&\left(Q_{\alpha} f\right)(x)=|x|^{2} f(x)-\lim _{N \rightarrow \infty} \frac{\mathrm{e}^{i a x}}{4 \pi N} \int_{D_{N}^{3}} \mathrm{e}^{-i a x} f(x) d^{3} x,
\end{aligned}\right. \\
& n=2, \quad\left\{\begin{aligned}
& \operatorname{dom}\left(Q_{\alpha}\right)=\left\{f \in L^{2}\left(\mathbb{R}^{2}\right) \mid Q_{\alpha} f \in L^{2}\left(\mathbb{R}^{2}\right),\right. \\
&\left.\int_{D_{N}^{2}} \mathrm{e}^{-i a x} f(x) d^{2} x=c\left(2 \pi \alpha \log N+\frac{\pi^{2}}{2}\right)+o(1), c \in \mathbb{C}\right\}, \\
&\left(Q_{\alpha} f\right)(x)=|x|^{2} f(x)-\lim _{N \rightarrow \infty} \frac{\mathrm{e}^{i a x}}{2 \pi \log N} \int_{B_{N}^{2}} \mathrm{e}^{-i a x} f(x) d^{2} x,
\end{aligned}\right. \\
& n=1, \quad\left\{\begin{aligned}
& \operatorname{dom}\left(Q_{\alpha}\right)=\left\{f \in L^{2}(\mathbb{R}) \mid Q_{\alpha} f \in L^{2}(\mathbb{R}),\right. \\
&\left.\int_{D_{N}^{1}} \mathrm{e}^{-i a x} f(x) d x=c\left(-\frac{2 \alpha}{N}+\frac{\pi \alpha}{\sqrt{2}}+\frac{\pi}{\sqrt{2}}\right)+o(1 / N), c \in \mathbb{C}\right\}, \\
&\left(Q_{\alpha} f\right)(x)=|x|^{2} f(x)-\frac{\sqrt{2} \alpha \mathrm{e}^{i a x}}{\pi(\alpha+1)} \int_{\mathbb{R}} \mathrm{e}^{-i a x} f(x) d x .
\end{aligned}\right.
\end{aligned}
$$

Lemma II.17 Let $Q_{\alpha}$ be any of the self adjoint extensions of the non maximal multiplication operator $\dot{Q}$ described in Lemma II.16. Then, for all $\lambda \in \rho\left(Q_{\alpha}\right) \cap \rho\left(Q_{0}\right), Q_{\alpha}$ has the resolvent

$$
\begin{array}{ll}
n=3, & R\left(\lambda, Q_{\alpha}\right) \phi=R\left(\lambda, Q_{0}\right) \phi+\frac{1}{\sqrt{2} \pi^{2}\left(\frac{1}{\alpha}-1-i \sqrt{2 \lambda}\right)} \int_{\mathbb{R}^{3}} \mathrm{e}^{-i a x}\left(R\left(\lambda, Q_{0}\right) \phi\right)(x) d^{3} x \psi_{\lambda}, \\
n=2, & R\left(\lambda, Q_{\alpha}\right) \phi=R\left(\lambda, Q_{0}\right) \phi+\frac{\pi^{2}}{\frac{\pi^{2}}{2 \alpha}+\pi \log (-\lambda)} \int_{\mathbb{R}^{2}} \mathrm{e}^{-i a x}\left(R\left(\lambda, Q_{0}\right) \phi\right)(x) d^{2} x \psi_{\lambda}, \\
n=1, & R\left(\lambda, Q_{\alpha}\right) \phi=R\left(\lambda, Q_{0}\right) \phi+\frac{1}{\frac{\pi}{\sqrt{2} \alpha}+\frac{\pi}{\sqrt{2}}-\frac{i \pi}{\sqrt{\lambda}}} \int_{\mathbb{R}} \mathrm{e}^{-i a x}\left(R\left(\lambda, Q_{0}\right) \phi\right)(x) d x \psi_{\lambda},
\end{array}
$$

where $\psi_{\lambda}(x)=\frac{\mathrm{e}^{i a x}}{|x|^{2}-\lambda}$ for all the cases.

Corollary II.18 The operator $R\left(\lambda, Q_{\alpha}\right)$ of Lemma II.17 is an integral operator with kernel

$$
\begin{array}{ll}
n=3, & \operatorname{ker}\left(x, y ; R\left(\lambda, Q_{\alpha}\right)\right)=\frac{\delta(x-y)}{\lambda-|x|^{2}}-\frac{1}{\sqrt{2} \pi^{2}\left(\frac{1}{\alpha}-1-i \sqrt{2 \lambda}\right)} \frac{\mathrm{e}^{i a(x-y)}}{\left(\lambda-|x|^{2}\right)\left(\lambda-|y|^{2}\right)}, \\
n=2, & \operatorname{ker}\left(x, y ; R\left(\lambda, Q_{\alpha}\right)\right)=\frac{\delta(x-y)}{\lambda-|x|^{2}}-\frac{\pi^{2}}{\frac{\pi^{2}}{2 \alpha}+\pi \log (-\lambda)} \frac{\mathrm{e}^{i a(x-y)}}{\left(\lambda-|x|^{2}\right)\left(\lambda|y|^{2}\right)}, \\
n=1, & \operatorname{ker}\left(x, y ; R\left(\lambda, Q_{\alpha}\right)\right)=\frac{\delta(x-y)}{\lambda-|x|^{2}}-\frac{\frac{1}{\sqrt{2} \alpha}+\frac{\pi}{\sqrt{2}}-\frac{i \pi}{\sqrt{\lambda}}}{\left(\lambda-|x|^{2}\right)\left(\lambda-\left.y\right|^{2}\right)},
\end{array}
$$

where $\lambda \in \rho\left(Q_{\alpha}\right) \cap \rho\left(Q_{0}\right)$ and $x, y \in \mathbb{R}^{n}, n=3,2,1$ respectively.

Moreover, we have: 
Lemma II.19 Let $Q_{\alpha}$ be any of the self adjoint extensions of the non maximal multiplication operator $\dot{Q}$ described in Lemma II.16, then the difference $R\left(\lambda, Q_{\alpha}\right)-R\left(\lambda, Q_{0}\right)$ is a trace-class operator and, assuming $\operatorname{Im} \sqrt{\lambda}>0$

$$
\begin{array}{ll}
n=3, & \operatorname{Tr}\left(R\left(\lambda, Q_{\alpha}\right)-R\left(\lambda, Q_{0}\right)\right)=\frac{1}{i \sqrt{2 \lambda}\left(\frac{1}{\alpha}-1-i \sqrt{2 \lambda}\right)}, \\
n=2, & \operatorname{Tr}\left(R\left(\lambda, Q_{\alpha}\right)-R\left(\lambda, Q_{0}\right)\right)=\frac{\pi}{\lambda} \frac{1}{\frac{\pi^{2}}{2 \alpha}+\pi \log (-\lambda)}, \\
n=1, & \operatorname{Tr}\left(R\left(\lambda, Q_{\alpha}\right)-R\left(\lambda, Q_{0}\right)\right)=\frac{i}{2 \lambda^{3 / 2}} \frac{1}{\frac{1}{\sqrt{2} \alpha}+\frac{1}{\sqrt{2}}-\frac{i}{\sqrt{\lambda}}} .
\end{array}
$$

Pooof. One has to compute the $L^{2}$-trace using the kernel given in Corollary $\amalg .18$ and the results of Lemma V.4 in the appendix.

\section{E. The basic example in the half space}

Let $\Omega^{n}=\mathbb{H}^{n}=[0, \infty) \times \mathbb{R}^{n-1}, e(x)=\sin (a x)$, where $a \in \mathbb{H}^{n}$, and $n=1,2,3$. We study in this section the self adjoint extensions of the (closure of the) operator $\dot{Q}$ in $L^{2}\left(\mathbb{H}^{n}\right)$ defined by

$$
\begin{aligned}
\operatorname{dom}(\dot{Q}) & =\left\{f \in L^{2}\left(\mathbb{H}^{n}\right) \mid q f \in L^{2}\left(\mathbb{H}^{n}\right), f\left(\{0\} \times \mathbb{R}^{n-1}\right)=0, \int_{\mathbb{H}^{n}} \sin (a x) f(x) d^{n} x=0\right\}, \\
\dot{Q} f & =q f
\end{aligned}
$$

where $q(x)=|x|^{2}$. We have $|e|=1$, and $B_{N}^{n}=D_{N}^{n} \cap \mathbb{H}^{n}$, and the functions $d_{n}(N)$ and $g_{n}(\lambda)$ appearing in equation (12) are given in Lemma $\mathrm{V} .3$ in the appendix.

Recalling that the space of the functions satisfying Dirichlet boundary condition on the boundary of the half space naturally identifies with the space of the odd functions on the whole space, we realize the delta function in the half space as

$$
\delta_{\mathbb{H}^{n}}(x-a)=\frac{1}{2}\left(\delta(x-a)-\delta\left(x-P_{n}(a)\right),\right.
$$

where $P_{n}$ is the reflection on the last coordinate.

Now we are able to write down explicitly all relevant quantities concernig the operator $\dot{Q}$ in the half-space $\mathbb{H}^{n}$ for $n=3,2,1$.

Assuming $\operatorname{Im} \sqrt{\lambda}>0$, from Lemma $\overline{V .3}$ in the appendix we have

$$
\begin{aligned}
& n=3, \quad\left\{\begin{array}{l}
\int_{B_{N}^{3}} \frac{\sin ^{2}(a x)}{|x|^{2}-\lambda} d^{3} x=\pi N+\frac{i \pi^{2}}{2} \sqrt{\lambda} \operatorname{sgn}(\operatorname{Im} \sqrt{\lambda})-\frac{\pi^{2}}{4 a} \mathrm{e}^{2 i a} \sqrt{\lambda} \operatorname{sgn}(\operatorname{Im} \sqrt{\lambda})+o(1), \\
\hat{d}_{3}(N)=\pi N, \quad \hat{g}_{3}(\lambda)=\frac{i \pi^{2}}{2} \sqrt{\lambda}-\frac{\pi^{2}}{4 a} \mathrm{e}^{2 i a} \sqrt{\lambda} \\
\hat{a}_{3}=\frac{\pi^{2}}{4}\left(\sqrt{2}+\frac{\mathrm{e}^{-\sqrt{2} a} \sin (\sqrt{2} a)}{2 a}\right), \quad \hat{b}_{3}=-\frac{\pi^{2}}{4}\left(\sqrt{2}+\frac{\mathrm{e}^{-\sqrt{2} a} \cos (\sqrt{2} a)}{2 a}\right),
\end{array}\right. \\
& n=2, \quad\left\{\begin{array}{l}
\int_{B_{N}^{2}} \frac{1}{|x|^{2}-\lambda} d^{2} x=\frac{\pi}{2} \log N-\frac{\pi}{4} \log (-\lambda)+\frac{\pi}{2} K_{0}(2 a \sqrt{-\lambda})+o(1), \\
\hat{d}_{2}(N)=\frac{\pi}{2} \log N, \quad \hat{g}_{2}(\lambda)=-\frac{\pi}{4} \log (-\lambda)+\frac{\pi}{2} K_{0}(2 a \sqrt{-\lambda}), \\
\hat{a}_{2}=\frac{\pi}{4}\left(\frac{\pi}{2}+i K_{0}(2 a \sqrt{i})-i K_{0}(2 a \sqrt{-i})\right), \quad b_{2}=-\frac{i \pi}{4}\left(K_{0}(2 a \sqrt{i})+K_{0}(2 a \sqrt{-i})\right),
\end{array}\right. \\
& n=1, \quad\left\{\begin{array}{l}
\int_{B_{N}^{1}} \frac{1}{|x|^{2}-\lambda} d x=\frac{i \pi}{4 \sqrt{\lambda} \operatorname{sgn}(\operatorname{Im} \sqrt{\lambda})}\left(1-\mathrm{e}^{2 i a \sqrt{\lambda} \operatorname{sgn}(\operatorname{Im} \sqrt{\lambda})}\right)-\frac{1}{2 N}+o\left(\frac{1}{N}\right), \\
\hat{d}_{1}(N)=-\frac{2}{N}, \quad \hat{g}_{1}(\lambda)=\frac{i \pi\left(1-\mathrm{e}^{2 i a \sqrt{\lambda}}\right)}{4 \sqrt{\lambda}}, \\
\hat{a}_{1}=\frac{\pi}{4 \sqrt{2}}\left(1-\mathrm{e}^{-\sqrt{2} a}(\cos (\sqrt{2} a)+\sin (\sqrt{2} a))\right), \quad \hat{b}_{1}=\frac{\pi}{4 \sqrt{2}}\left(1-\mathrm{e}^{-\sqrt{2} a}(\cos (\sqrt{2} a)-\sin (\sqrt{2} a))\right),
\end{array}\right.
\end{aligned}
$$

$K_{0}(z)$ being a Bessel function. Here we use the "hat" to distinguish the latter quantities with respect to the ones appearing in the example discussed in Section IID concerning the case of the whole space.

Now, applying Propositions $\amalg .9$ and II.11 and Corollary I.13, we prove the following results. 
Lemma II.20 The self adjoint extensions of $\dot{Q}$ are:

$$
\begin{aligned}
& n=3, \quad\left\{\begin{aligned}
& \operatorname{dom}\left(Q_{\alpha}\right)=\{ f \in L^{2}\left(\mathbb{R}^{3}\right) \mid Q_{\alpha} f \in L^{2}\left(\mathbb{R}^{3}\right), f\left(\{0\} \times \mathbb{R}^{2}\right)=0, \\
&\left.\int_{B_{N}^{3}} \sin (a x) f(x) d^{3} x=c\left(\pi N \alpha+\alpha \hat{b}_{3}+\hat{a}_{3}\right)+o(1), c \in \mathbb{C}\right\}, \\
& Q_{\alpha} f=|x|^{2} f(x)-\lim _{N \rightarrow \infty} \frac{\sin (a x)}{\pi N} \int_{B_{N}^{3}} \sin (a x) f(x) d^{3} x,
\end{aligned}\right. \\
& n=2, \quad\left\{\begin{aligned}
& \operatorname{dom}\left(Q_{\alpha}\right)=\left\{f \in L^{2}\left(\mathbb{H}^{2}\right) \mid Q_{\alpha} f \in L^{2}\left(\mathbb{H}^{2}\right), f(\{0\} \times \mathbb{R})=0,\right. \\
&\left.\int_{B_{N}^{2}} \sin (a x) f(x) d^{2} x=c\left(\frac{\pi \alpha \log N}{2}+\alpha \hat{b}_{2}+\hat{a}_{2}\right)+o(1), c \in \mathbb{C}\right\}, \\
&\left(Q_{\alpha} f\right)(x)=|x|^{2} f(x)-\lim _{N \rightarrow \infty} \frac{2 \sin (a x)}{\pi \log N} \int_{B_{N}^{2}} \sin (a x) f(x) d^{2} x,
\end{aligned}\right. \\
& n=1, \quad\left\{\begin{aligned}
& \operatorname{dom}\left(Q_{\alpha}\right)=\left\{f \in L^{2}(R) \mid Q_{\alpha} f \in L^{2}(R), f(0)=0,\right. \\
&\left.\int_{B_{N}^{1}} \sin (a x) f(x) d x=c\left(-\frac{2 \alpha}{N}+\alpha \hat{b}_{1}+\hat{a}_{1}\right)+o(1 / N), c \in \mathbb{C}\right\}, \\
&\left(Q_{\alpha} f\right)(x)=|x|^{2} f(x)-\frac{4 \sqrt{2} \alpha \sin (a x) \int_{\mathrm{H}^{1}} \sin (a x) f(x) d x}{\pi(1+\alpha)\left(1-\mathrm{e}^{-\sqrt{2} a} \cos (\sqrt{2} a)\right)-\pi(1-\alpha) \mathrm{e}^{-\sqrt{2} a} \sin (\sqrt{2} a)} .
\end{aligned}\right.
\end{aligned}
$$

Lemma II.21 Let $Q_{\alpha}$ be any of the self adjoint extensions of the non maximal multiplication operator $\dot{Q}$ described in Lemma II.20. Then, for all $\lambda \in \rho\left(Q_{\alpha}\right) \cap \rho\left(Q_{0}\right), Q_{\alpha}$ has the resolvent (we assume $\operatorname{Im} \sqrt{\lambda}$ to be positive)

$$
\begin{aligned}
& n=3, \quad R\left(\lambda, Q_{\alpha}\right) \phi=R\left(\lambda, Q_{0}\right) \phi+\frac{\int_{\mathrm{H}^{3}} \sin (a x)\left(R\left(\lambda, Q_{0}\right) \phi\right)(x) d^{3} x}{\frac{\hat{a}_{3}}{\alpha}+\hat{b}_{3}-\left(\frac{i \pi^{2} \sqrt{\lambda}}{2}-\frac{\pi^{2} \mathrm{e}^{2 i a \sqrt{\lambda}}}{4 a}\right)} \psi_{\lambda}, \\
& n=2, \quad R\left(\lambda, Q_{\alpha}\right) \phi=R\left(\lambda, Q_{0}\right) \phi+\frac{\int_{\mathrm{H}^{2}} \sin (a x)\left(R\left(\lambda, Q_{0}\right) \phi\right)(x) d^{2} x}{\frac{\hat{a}_{2}}{\alpha}+\hat{b}_{2}+\frac{\pi \log (-\lambda)}{4}-\frac{\pi K_{0}(2 a \sqrt{-\lambda})}{2}} \psi_{\lambda}, \\
& n=1, \quad R\left(\lambda, Q_{\alpha}\right) \phi=R\left(\lambda, Q_{0}\right) \phi+\frac{\int_{\mathrm{H}^{1}} \sin (a x)\left(R\left(\lambda, Q_{0}\right) \phi\right)(x) d x}{\frac{\hat{a}_{1}}{\alpha}+\hat{b}_{1}-\frac{i \pi\left(1-\mathrm{e}^{2 i a} \sqrt{\lambda}\right)}{4 \sqrt{\lambda}}} \psi_{\lambda},
\end{aligned}
$$

where $\psi_{\lambda}(x)=\frac{\sin (a x)}{|x|^{2}-\lambda}$ for all the cases.

Corollary II.22 The operator $R\left(\lambda, Q_{\alpha}\right)$ of Lemma II.21 is an integral operator with kernel

$$
\begin{aligned}
& n=3, \quad \operatorname{ker}\left(x, y ; R\left(\lambda, Q_{\alpha}\right)\right)=\frac{\delta_{\mathrm{H} 3}(x-y)}{\lambda-|x|^{2}}-\frac{1}{\frac{\hat{a}_{3}}{\alpha}+\hat{b}_{3}-\left(\frac{i \pi^{2} \sqrt{\lambda}}{2}-\frac{\pi^{2} \mathrm{e}^{2 i a} \sqrt{\lambda}}{4 a}\right)} \frac{\sin (a x) \sin (a y)}{\left(\lambda-|x|^{2}\right)\left(\lambda-|y|^{2}\right)}, \\
& n=2, \quad \operatorname{ker}\left(x, y ; R\left(\lambda, Q_{\alpha}\right)\right)=\frac{\delta_{\mathrm{H}^{2}}(x-y)}{\lambda-|x|^{2}}-\frac{1}{\frac{\hat{a}_{2}}{\alpha}+\hat{b}_{2}+\frac{\pi \log (-\lambda)}{4}-\frac{\pi K_{0}(2 a \sqrt{-\lambda})}{2}} \frac{\sin (a x) \sin (a y)}{\left(\lambda-|x|^{2}\right)\left(\lambda-|y|^{2}\right)}, \\
& n=1, \quad \operatorname{ker}\left(x, y ; R\left(\lambda, Q_{\alpha}\right)\right)=\frac{\delta_{\mathrm{H} 1}(x-y)}{\lambda-|x|^{2}}-\frac{1}{\frac{\hat{a}_{1}}{\alpha}+\hat{b}_{1}-\frac{i \pi\left(1-\mathrm{e}^{2 i a \sqrt{\lambda}}\right)}{4 \sqrt{\lambda}}} \frac{\sin (a x) \sin (a y)}{\left(\lambda-|x|^{2}\right)\left(\lambda-|y|^{2}\right)},
\end{aligned}
$$

where $\lambda \in \rho\left(Q_{\alpha}\right) \cap \rho\left(Q_{0}\right), x, y \in \mathbb{H}^{n}, n=3,2,1$.

Moreover, we have:

Lemma II.23 Let $Q_{\alpha}$ be any of the self adjoint extensions of the non maximal multiplication operator $\dot{Q}$ described in Lemma II.16, then the difference $R\left(\lambda, Q_{\alpha}\right)-R\left(\lambda, Q_{0}\right)$ is a trace-class operator and, assuming $\operatorname{Im} \sqrt{\lambda}>0$

$$
\begin{aligned}
& n=3, \quad \operatorname{Tr}\left(R\left(\lambda, Q_{\alpha}\right)-R\left(\lambda, Q_{0}\right)\right)=\frac{\pi^{2}\left(1-\mathrm{e}^{2 i a \sqrt{\lambda}}\right)}{2 i \sqrt{\lambda}} \frac{1}{\frac{\hat{a}_{3}}{\alpha}+\hat{b}_{3}-\left(\frac{i \pi^{2} \sqrt{\lambda}}{2}-\frac{\pi^{2} \mathrm{e}^{2 i a \sqrt{\lambda}}}{4 a}\right)}, \\
& n=2, \quad \operatorname{Tr}\left(R\left(\lambda, Q_{\alpha}\right)-R\left(\lambda, Q_{0}\right)\right)=\frac{\pi\left(1+2 i a K_{1}(-2 i a \sqrt{\lambda})\right)}{2 \lambda} \frac{1}{\frac{\hat{a}_{2}}{\alpha}+\hat{b}_{2}+\frac{\pi \log (-\lambda)}{4}-\frac{\pi K_{0}(2 a \sqrt{-\lambda})}{2}}, \\
& n=1, \quad \operatorname{Tr}\left(R\left(\lambda, Q_{\alpha}\right)-R\left(\lambda, Q_{0}\right)\right)=\left(\frac{i \pi\left(1-\mathrm{e}^{2 i a \sqrt{\lambda}}\right)}{4 \lambda^{3 / 2}}-\frac{\pi a \mathrm{e}^{2 i a \sqrt{\lambda}}}{2 \lambda}\right) \frac{1}{\frac{\hat{a}_{1}}{\alpha}+\hat{b}_{1}-\frac{i \pi\left(1-\mathrm{e}^{2 i a \sqrt{\lambda}}\right)}{4 \sqrt{\lambda}}},
\end{aligned}
$$

where $K_{1}$ is a Bessel function.

Pooof. All latter integrals are computed in Lemma $\mathrm{V.5}$ in the appendix. 


\section{THE LAPLACE OPERATOR WITH DELTA POTENTIAL}

We show in this section how the extensions of the multiplication operators introduced in Section $\amalg \mathrm{C}$ are used in order to define a self adjoint differential operator corresponding to the formal Laplacian operator with a delta type potential, discussed in Section [ This was the original approach of Berezin and Fadeev [5]. Let

$$
-d=-\sum_{j=1}^{n} \frac{\partial^{2}}{\partial x_{j}^{2}},
$$

denotes the formal Laplace operator on $\Omega^{n}$, where $\Omega^{n}$ is either $\mathbb{R}^{n}$ or $\mathbb{H}^{n}$. Let $a$ be a point in $\Omega^{n}$ and $\mathcal{F}$ denotes the Fourier transform in $\Omega^{n}$. Then, the operator $-\dot{\Delta}=\mathcal{F}^{-1} \dot{Q} \mathcal{F}$, is a (closed) symmetric operator in $L^{2}\left(\Omega^{n}\right)$, with deficiency indices $(1,1)$, for $n \leq 3$, and domain

$$
\operatorname{dom}(-\dot{\Delta})=\left\{f \in W^{2,2}\left(\Omega^{n}\right) \mid f(a)=0\right\}, \quad-\dot{\Delta} f=-d f
$$

unitary equivalent to the operator $\dot{Q}$ defined at the beginning of Section $\amalg$, with $e(x)$ either $\mathrm{e}^{i a x}$ when $\Omega^{n}=\mathbb{R}^{n}$ or $\sin (a x)$ when $\Omega^{n}=\mathbb{H}^{n}$. This follows immediately in both cases by the definition of the Fourier transform. Therefore, all the self adjoint extensions of $-\dot{\Delta}$ are the operators $-\Delta_{\alpha}=\mathcal{F}^{-1} Q_{\alpha} \mathcal{F}$, where the operators $Q_{\alpha}$ were defined in general in Proposition [I.9, and in the particular case of dimensions $n=3,2$ and 1 in Lemmas ЏI.16 and 【I.20 respectively when $\Omega^{n}=\mathbb{R}^{n}$ or $R^{n}$. In all cases, the maximal operator is $-\Delta_{0}$ with

$$
\operatorname{dom}\left(-\Delta_{0}\right)=W^{2,2}, \quad-\Delta_{0} f=-d f
$$

It is worth to observe here that the operator $\dot{\Delta}$ can also be introduced directly (this is the approach of [1]) as the closure of the operator

$$
\operatorname{dom}(-\tilde{\Delta})=C_{0}^{\infty}\left(\Omega^{n}-\{a\}\right), \quad-\tilde{\Delta} f=-d f .
$$

This follows adapting the standard proof for the maximal operators (see for example [31] 10.11). For it is clear that if $f \in \operatorname{dom}(-\dot{\Delta})$, then $f(a)=\left(\mathcal{F}^{-1} \mathcal{F} f\right)(a)=0$, and therefore $f \in \operatorname{dom}(\dot{Q})$. Conversely, given $f \in \operatorname{dom}(\dot{Q})$, we have that $\left(\mathcal{F}^{-1} f\right)(a)=0$. Since the closure of the restriction of $\dot{Q}$ on $C_{0}^{\infty}\left(\Omega^{n}\right)$ is closed, given a sequence $\left\{h_{n}\right\}$ in $C_{0}^{\infty}\left(\Omega^{n}\right)$ such that $h_{n} \rightarrow h=\mathcal{F}^{-1} f$, and $\dot{Q} h_{n} \rightarrow \dot{Q} h$, the condition $h(a)=0$ can only be satisfied if the functions $h_{n}$ have support away from $a$.

Next we interpret the operators $-\Delta_{\alpha}$ as a perturbation of the maximal operator $-\Delta_{0}$, using the theory of singular perturbation developed in Section 1 of [3], that we recall here briefly. Let $A$ be a self adjoint operator in the Hilbert space $\mathcal{H}$ as in Section IA Let $\gamma$ be a real number and $a \in \mathcal{H}_{-2}$ with norm 1. A singular rank one perturbation of the operator $A$ is the operator defined by the following formula

$$
A^{\gamma}=A+\gamma \mathrm{a}_{c}(\cdot) \mathrm{a}
$$

where $\mathrm{a}_{c}$ is either a or a linear bounded extension of a [3] Section 1.3.2, depending whether a $\in \mathcal{H}_{-1}-\mathcal{H}$ or a $\in \mathcal{H}_{-2}-\mathcal{H}_{-1}$. A rigorous definition of this type of operators acting on the dual space of functionals $\mathcal{H}_{-2}$ has been given in [3] Section 1.3. The domain and the action of the operator are described in Theorems 1.3.1 and 1.3.2, respectively. Using this approach, we can define singular perturbed operators in the Hilbert space $\mathcal{H}$, by taking the restrictions of the operators $A^{\gamma}$ just defined (see equation (1.45) of [3] for the domain). We will use this definition and we will use the same notation. The operators defined in this way are self adjoint.

If $\dot{A}$ is a symmetric operator in $\mathcal{H}$ with deficiency indices $(1,1)$, as in Section $\amalg \mathrm{A}$, the self adjoint extension $A_{\alpha}$ of $\dot{A}$ described in Proposition $\llbracket .3$, with $\alpha=\operatorname{ctg} \theta$ (or in Proposition $\llbracket .9$, when $\dot{A}=\dot{Q}$ ), coincides with the singular rank one perturbation $A^{\gamma}$ of the operator $A_{0}$ if

$$
\frac{1}{\gamma}=\frac{1}{\alpha}-c
$$

by Theorem 1.3.3 and the results of Section 1.3 of [3], and where $c$ is a real number. Now, the mapping

$$
\mathrm{d}: f \mapsto \int_{\Omega^{n}} \delta_{\Omega^{n}}(x-a) f(x) d^{n} x
$$


defines a functional on $\mathcal{H}_{2}=W^{2,2}\left(\Omega^{n}\right)$, and it is easy to see that $\mathrm{d} \in \mathcal{H}_{-2}-\mathcal{H}_{-1}$ (see also [3] Section 1.5.1). However, $\|\mathrm{d}\|_{-2} \neq 1$, thus we need to take in account a normalization factor and we define $\mathrm{a}=\frac{\mathrm{d} \|_{-2}}{\|\mathrm{~d}\|_{-2}}\left(\right.$ note that $\left.\|\mathrm{d}\|_{-2} \neq 0\right)$. Since

$$
\mathrm{a}(f)=\frac{f(a)}{\|\mathrm{d}\|_{-2}},
$$

the operator $-\Delta_{\alpha}$ corresponds to the singular rank one perturbation of the operator $-\Delta_{0}=-\Delta^{0}$

$$
-\Delta^{\gamma}=-\Delta^{0}+\gamma \mathrm{a}_{c}(\cdot) \mathrm{a}=-\Delta^{0}+\frac{\gamma}{\|\mathrm{d}\|_{-2}^{2}} \mathrm{~d}_{c}(\cdot) \mathrm{d},
$$

if we take $\frac{1}{\gamma}=\frac{1}{\alpha}-c$, for any real $c$. If we compare this with the formal regularization $-\Delta^{g_{R}}=-\Delta+g_{R} \delta$, of the formal perturbed operator $-\Delta^{g}=-\Delta^{0}+g \delta$ introduced in Section प (see equations (3) and (5)), we get $g_{R}=\frac{\gamma}{\|\mathrm{d}\|_{-2}^{2}}$ and hence

$$
\frac{\|\mathrm{d}\|_{-2}^{2}}{\alpha}=\frac{1}{g_{R}}+c\|\mathrm{~d}\|_{-2}^{2} .
$$

Summing up, we have proved that the regularized formal operator $-\Delta^{g_{R}}=-\Delta+g_{R} \delta$, describing the Laplace operator with a delta type interaction considered in the introduction, corresponds to the operator $-\Delta^{\gamma}=-\Delta_{\alpha=\frac{\gamma}{1+c \gamma}}$, with $\gamma=\|\mathrm{d}\|_{-2}^{2} g_{R}$, and with any real $c$, and therefore it is unitary equivalent to the operator $Q_{\alpha=\frac{\gamma}{1+c \gamma}}$, defined explicitly in Sections IID and IIE, respectively when $\Omega^{n}=\mathbb{R}^{n}$ or $\mathbb{H}^{n}$. The resolvents are given by taking Fourier transform of the resolvents given in Lemmas II.17 and II.21, In all cases, the operator $-\Delta^{0}$ is the maximal operator, and the difference of the resolvents $R\left(\lambda,-\Delta^{g_{R}}\right)-R\left(\lambda,-\Delta^{0}\right)$ is of trace class. The trace is given in Lemmas $\amalg .19$ and II.23.

In particular, we use this result in the formula for the difference of the resolvents given in Proposition II.11. Since e is the Fourier transform of $\mathrm{d},\|\mathrm{d}\|_{-2}^{2}=\|\mathrm{e}\|_{-2}^{2}=\left\|\psi_{+}\right\|_{0}^{2}=a_{n}$, we obtain

$$
\left(R\left(\lambda,-\Delta^{g_{R}}\right)-R\left(\lambda,-\Delta^{0}\right)\right) \phi=\frac{1}{\frac{1}{g_{R}}+a_{n} c+b_{n}-g_{n}(\lambda)} \int_{\Omega^{n}} \bar{e}(x)\left(R\left(\lambda,-\Delta^{0}\right) \phi\right)(x) d^{n} x \psi_{\lambda} .
$$

As observed in [3], we have two free constants in this formula, and therefore a two parameters family of operators. While the constant $g_{R}$ has a physical meaning, since it is the coupling constant discussed in Section \, the constant $c$ should be fixed. However, a prescription to fix the constant $c$ has been introduced in Section 1.3.3 of [3] for the class of the homogeneous operators, defined as follows. Suppose there exists a group $G$ of unitary transformations of the Hilbert space $\mathcal{H}$. An operator $A$ is said to be homogeneous if it rescales in an appropriate way under the action of $G$, as in Lemma 1.3.2 of [3]. Now, suppose the operator $A^{0}$ is homogeneous accordingly to this definition. If this is the case, in the same lemma a condition is given for the existence of a singular rank one perturbation $A^{\gamma}$ of $A^{0}$, and its unicity is proved. The proof is based on the fact that the self adjoint operator $A^{\gamma}$ satisfies the same symmetry property as $A^{0}$ for one and only one value of the constant $c$. This condition fixes the value of $c$. The case of the Laplace operator $-\Delta$ in $\mathbb{R}^{3}$ was discussed in Section 1.5.5 of [3], where it is shown that $-\Delta$ is an homogeneous operator with respect to the group of the scaling transformations of $L^{2}\left(\mathbb{R}^{3}\right)$. We review this case and we also investigate the one dimensional case in the following Section IV B. However, as observed at the end of Section 1.3.3 of [3], in the 2-dimensional case, the Laplace operator is homogeneous but the condition for the existence of the singular rank one perturbation is not satisfied. It follows that singular rank one perturbations of the Laplace operator in dimension two do not exist.

The situation is more difficult for the case of the Hilbert space $L^{2}\left(\mathbb{H}^{n}\right), n=1,2,3$. For in this case we do not have the group of symmetry given by scaling transformations, and consequently the prescription described above does not apply. On the other side, the situation is more delicate because of the following reason. If we compare the formula for the resolvent given in equation (15) with the heuristic formula given in equation (5), a straightforward calculation shows that the two coincide if and only if we identify

$$
\frac{1}{g_{R}}=\frac{1}{g_{R}}+a_{n} c+b_{n}
$$

This condition can be satisfied either re-regularizing the coupling constant, or assuming the condition $c=-\frac{b_{n}}{a_{n}}$. Since the constants $a_{n}$ and $b_{n}$ depend on the geometry through the parameter $a$, the first possibility contradicts the plausible physical requirement that the coupling constant should not depend on the geometry. Therefore, we will assume the second possibility. With this choice, the self adjoint extension associated to the coupling constant $g_{R}$ is characterized in the following proposition and its corollary, whose proofs follow by the results of the previous sections. 
Proposition III.1 The operator $-\Delta^{g_{R}}$ is the self adjoint extension of the non maximal multiplication operator $-\dot{\Delta}$ with resolvent

$$
\begin{aligned}
R\left(\lambda,-\Delta^{g_{R}}\right) \phi & =R\left(\lambda,-\Delta^{0}\right) \phi+\frac{1}{\frac{1}{g_{R}}-g_{n}(\lambda)} \int_{\Omega^{n}} \bar{e}(x)\left(R\left(\lambda,-\Delta^{0}\right) \phi\right)(x) d^{n} x \psi_{\lambda}, \\
\psi_{\lambda}(x) & =\frac{e(x)}{|x|^{2}-\lambda},
\end{aligned}
$$

where $\lambda \in \rho\left(-\Delta^{g_{R}}\right) \cap \rho\left(-\Delta^{0}\right)$, and either $e(x)=\mathrm{e}^{i a x}$ and the functions $g_{n}(\lambda)$ are given for $n=3,2,1$ and $\Omega^{n}=\mathbb{R}^{n}$ in Section IID or $e(x)=\sin ($ ax $)$ and the $g_{n}(\lambda)$ are given for $n=3,2,1$ and $\Omega^{n}=\mathbb{H}^{n}$ in Section IIE. We assume $g_{R} g_{n}(\lambda) \neq 1$, which corresponds to pure continuum spectrum (see Lemma III.4).

Corollary III.2 The difference of the resolvent $R\left(\lambda,-\Delta^{g_{R}}\right)-R\left(\lambda,-\Delta^{0}\right)$ is trace class with trace

$$
\operatorname{Tr}\left(R\left(\lambda,-\Delta^{g_{R}}\right)-R\left(\lambda,-\Delta^{0}\right)\right)=-\frac{1}{\frac{1}{g_{R}}-g_{n}(\lambda)} \int_{\Omega^{n}} \frac{|e(x)|^{2}}{\left(|x|^{2}-\lambda\right)^{2}} d^{n} x .
$$

Remark III.3 In other words, we obtain formulas for the trace of the difference of the resolvents $\operatorname{Tr}\left(R\left(\lambda,-\Delta^{g_{R}}\right)-R\left(\lambda,-\Delta^{0}\right)\right)$ simply by taking the correspondent formulas given in Lemmas II.19 and II.23 and making the substitution $\frac{1}{g_{R}}=\frac{1}{\frac{a_{n}}{\alpha}+b_{n}}$, or $\frac{1}{g_{R}}=\frac{1}{\frac{a_{n}}{\alpha}+\hat{b}_{n}}$, respectively.

Explicit formulas for the trace for the cases of interest will be given in the following Section IV

We conclude this section by studying the eigenvalues of the operators $-\Delta^{g_{R}}$.

Lemma III.4 Let $-\Delta^{g_{R}}$ be the operator with resolvent given in Proposition [II.1. Then, the point spectrum of $-\Delta^{g_{R}}$, $\operatorname{Sp}_{p}\left(-\Delta^{g_{R}}\right)=\operatorname{Sp}_{d}\left(-\Delta^{g_{R}}\right)$, is given as follows:

- if $\Omega^{n}=\mathbb{R}^{3}$ or $\Omega^{n}=\mathbb{R}^{1}$, then $\operatorname{Sp}_{p}\left(-\Delta^{g_{R}}\right)=\emptyset$ if $g_{R} \geq 0$, while there is one negative eigenvalue otherwise;

- if $\Omega^{n}=\mathbb{H}^{3}$, then $\operatorname{Sp}_{p}\left(-\Delta^{g_{R}}\right)=\emptyset$ if $a \geq-\frac{2 g_{R}}{\pi^{2}}$, while there is one negative eigenvalue otherwise;

- if $\Omega^{n}=\mathbb{H}^{1}$, we distinguish two cases: if $g_{R}$ is finite, then $\operatorname{Sp}_{p}\left(-\Delta^{g_{R}}\right)=\emptyset$ if $a \leq \frac{2 g_{R}}{\pi}$, while there is one negative eigenvalue otherwise; if $g_{R}=\infty$, then $\operatorname{Sp}_{p}\left(-\Delta^{g_{R}}\right)=\left\{\frac{\pi^{2} k^{2}}{a^{2}}\right\}_{k \in \mathbb{Z}_{0}}$.

Pooof. Assume $\operatorname{Im} \sqrt{\lambda} \geq 0$. Consider first the case of $\Omega^{n}=\mathbb{R}^{n}$. Then, the first statement follow from Theorems 1.1.4 and 3.1.4 of [1].

Next, consider the case of $\Omega^{n}=\mathbb{H}^{n}$. If $n=3$, the possible eigenvalues are the solutions of the equation

$$
\frac{1}{g_{R}}-\frac{\pi^{2}}{2} i \sqrt{\lambda}+\frac{\pi^{2}}{4 a} \mathrm{e}^{2 i a \sqrt{\lambda}}=0
$$

Let $\sqrt{\lambda}=x+i y$. Then, equation (16) becomes

$$
b-i(x+i y)+\frac{\mathrm{e}^{2 i a(x+i y)}}{2 a}=b+y-i x+\frac{\mathrm{e}^{-2 a y}}{2 a}(\cos (2 a x)+i \sin (2 a x))=0,
$$

with $b=\frac{2}{\pi^{2} g_{R}}$, and separating the real and imaginary parts

$$
\left\{\begin{array}{l}
b+y+\frac{\mathrm{e}^{-2 a y}}{2 a} \cos (2 a x)=0, \\
x-\frac{\mathrm{e}^{-2 a y}}{2 a} \sin (2 a x)=0 .
\end{array}\right.
$$

Since $\lambda=x^{2}-y^{2}+2 i x y$ must be real, $\sqrt{\lambda}=x+i y$ must be purely real or purely imaginary. Thus we look for solutions with $x=0$ or $y=0$. In the first case, $x=0$, the system in equation (17) reduces to the equation

$$
y=-b-\frac{\mathrm{e}^{-2 a y}}{2 a}
$$


that can be solved graphically with $f_{1}(y)=y, f_{2}(y)=-b-\frac{\mathrm{e}^{-2 a y}}{2 a}$. Since we have assumed $y \geq 0$, the existence of solutions depends on the value of $f_{2}(0)$. If $f_{2}(0)<0$, then there are no solutions. Since

$$
f_{2}(0)=-b-\frac{1}{2 a}
$$

and this quantity is negative for all $a$ if $g_{R} \geq-\frac{2 a}{\pi^{2}}$, it follows that there are no solutions for all $a$ and $g_{R} \geq-\frac{2 a}{\pi^{2}}$, and there is one negative eigenvalue otherwise.. In the second case, $y=0$, and the system becomes

$$
\left\{\begin{array}{l}
b+\frac{1}{2 a} \cos (2 a x)=0, \\
x-\frac{1}{2 a} \sin (2 a x)=0,
\end{array}\right.
$$

that has only the trivial solution, $x=0(b=-2 / 2 a)$.

If $n=2$, the possible eigenvalues are the solutions of the equation

$$
\frac{1}{g_{R}}-\frac{\pi}{4} \log (-\lambda)+\frac{\pi}{2} K_{0}(2 a \sqrt{\lambda})=0 .
$$

If $n=1$, the possible eigenvalues are the solutions of the equation

$$
\frac{1}{g_{R}}-\frac{\pi i}{4 \sqrt{\lambda}}\left(1-\mathrm{e}^{2 i a \sqrt{\lambda}}\right)=0
$$

With $b=\frac{4}{g_{R} \pi}$, equation (19) becomes

$$
i b \sqrt{\lambda}+1-\mathrm{e}^{2 i a \sqrt{\lambda}}=0,
$$

that gives the system

$$
\left\{\begin{array}{l}
1-b y-\mathrm{e}^{-2 a y} \cos (2 a x)=0, \\
b x-\mathrm{e}^{-2 a y} \sin (2 a x)=0 .
\end{array}\right.
$$

With $x=0$, we obtain

$$
y=\frac{1}{b}-\frac{\mathrm{e}^{-2 a y}}{b} .
$$

Since $f_{2}(y)=\frac{1}{b}-\frac{\mathrm{e}^{-2 a y}}{b}$ has tangent with angular coefficient $f_{2}^{\prime}(0)=\frac{2 a}{b}$, the system in equation (20) has one positive solution if and only if $a>\frac{b}{2}$. With $y=0$, the system in equation (20) becomes

$$
\left\{\begin{array}{l}
1-\cos (2 a x)=0 \\
b x-\sin (2 a x)=0
\end{array}\right.
$$

This system has only the trivial solution $x=0$ if $b \neq 0$, and has infinitely many solutions $x=\frac{\pi k}{a}, k \in \mathbb{Z}$, if $b=0$.

We point out that the spectrum in the case $\mathbb{H}^{1}=[0, \infty), g_{R}=\infty$, is as expected, since in this case the operator reduces to the sum of the Laplacian on the positive half line with Dirichlet boundary conditions, plus the Laplacian on the interval $[0, a]$.

\section{DETERMINANT AND PARTITION FUNCTIONS}

The aim of this section is to study the determinant of the operators described in Section III, and consequently to obtain explicit expression for the partition function of the associated models of the Casimir effect. We first recall how the zeta function regularization [19] (see, also [12] and references therein) is used to define the infinite determinants of self adjoint positive operators $A$. In fact, one defines

$$
\operatorname{det}_{\zeta} A=\mathrm{e}^{-\zeta^{\prime}(0, A)},
$$


where the zeta function of $A$ is by definition

$$
\zeta(s, A)=\sum_{\lambda \in \operatorname{Sp} A} \lambda^{-s}
$$

for $\operatorname{Re}(s)$ sufficiently large, and analytically continued elsewhere. Accordingly, we have for the the partition function $Z=\left(\operatorname{det}_{\zeta} \ell^{2} A\right)^{-\frac{1}{2}}$,

$$
\log Z=\frac{1}{2} \zeta^{\prime}(0, A)-\frac{1}{2} \zeta(0, A) \log \ell^{2},
$$

where $\ell$, a real non vanishing number, is the usual renormalization parameter.

More precisely, we need relative zeta functions and relative zeta determinants. We recall first the main definitions and some properties of relative zeta determinants in Section IVA and then we apply this method to the operators of interest in Section IVB.

\section{A. Relative zeta determinant and relative partition function}

We will use the notation introduced in [30] for relative zeta functions and we refer to that work or to the original paper of W. Müller for more details [24].

Let $\mathcal{H}$ be a separable Hilbert space, and let $A$ and $A_{0}$ be two self adjoint non negative linear operators in $\mathcal{H}$. Suppose that $\operatorname{Sp} A=\operatorname{Sp}_{c} A$, namely that $A$ has a pure continuous spectrum. We recall that $R(\lambda, T)=(\lambda I-T)^{-1}$ denotes the resolvent of the operator $T$, and $\rho(T)$ the resolvent set. Then, we introduce the following set of conditions on the pair $\left(A, A_{0}\right)$ :

(B.1) the operator $R(\lambda, A)-R\left(\lambda, A_{0}\right)$ is of trace class for all $\lambda \in \rho(A) \cap \rho\left(A_{0}\right)$;

(B.2) as $\lambda \rightarrow \infty$ in $\rho(A) \cap \rho\left(A_{0}\right)$, there exists an asymptotic expansion of the form:

$$
\operatorname{Tr}\left(R(\lambda, A)-R\left(\lambda, A_{0}\right)\right) \sim \sum_{j=0}^{\infty} \sum_{k=0}^{K_{j}} a_{j, k}(-\lambda)^{\alpha_{j}} \log ^{k}(-\lambda),
$$

where $-\infty<\cdots<\alpha_{1}<\alpha_{0}, \alpha_{j} \rightarrow-\infty$, for large $j$, and $a_{j, k}=0$ for $k>0 ;$

(B.3) as $\lambda \rightarrow 0$, there exists an asymptotic expansion of the form

$$
\operatorname{Tr}\left(R(\lambda, A)-R\left(\lambda, A_{0}\right)\right) \sim \sum_{j=0}^{\infty} b_{j}(-\lambda)^{\beta_{j}}
$$

where $-1 \leq \beta_{0}<\beta_{1}<\ldots$, and $\beta_{j} \rightarrow+\infty$, for large $j$.

We introduce the further consistency condition

(C) $\alpha_{0}<\beta_{0}$.

It was proved in [30] that if the pair of non negative self adjoint operators $\left(A, A_{0}\right)$ satisfies conditions (B.1)-(B.3), then it satisfies the conditions (1.1)-(1.3) of [24]. In this situation we define the relative zeta function for the pair $\left(A, A_{0}\right)$ by the following equation

$$
\zeta\left(s ; A, A_{0}\right)=\frac{1}{\Gamma(s)} \int_{0}^{\infty} t^{s-1} \operatorname{Tr}\left(\mathrm{e}^{-t A_{c}}-\mathrm{e}^{-t A_{0}}\right) d t,
$$

when $\alpha_{0}+1<\operatorname{Re}(s)<\beta_{0}+1$, and by analytic continuation elsewhere, and we define the regularized relative determinant of the pair of operators $\left(A, A_{0}\right)$ by

$$
\operatorname{det}_{\zeta}\left(A, A_{0}\right)=\mathrm{e}^{-\left.\frac{d}{d s} \zeta\left(s ; A, A_{0}\right)\right|_{s=0}} .
$$
[30].

Introducing the relative spectral measure, we have the following useful representation of the relative zeta function 
Proposition IV.1 Let $A$ be a non negative self adjoint operator and assume that there exists an operator $A_{0}$ such that the pair $\left(A, A_{0}\right)$ satisfies conditions (B.1)-(B.3), and $(C)$. Then,

$$
\zeta\left(s ; A, A_{0}\right)=\int_{0}^{\infty} v^{-2 s} e\left(v ; A, A_{0}\right) d v,
$$

where the relative spectral measure is defined by

$$
\begin{aligned}
& e\left(v ; A, A_{0}\right)=\frac{v}{\pi i} \lim _{\epsilon \rightarrow 0^{+}}\left(r\left(v^{2} \mathrm{e}^{2 i \pi-i \epsilon} ; A, A_{0}\right)-r\left(v^{2} \mathrm{e}^{i \epsilon} ; A, A_{0}\right)\right), \\
& r\left(\lambda ; A, A_{0}\right)=\operatorname{Tr}\left(R(\lambda, A)-R\left(\lambda, A_{0}\right)\right) .
\end{aligned}
$$

The integral, the limit and the trace exist.

Accordingly, we also define the zeta regularized partition function of a model described by the operator $A$, under the assumption that there exists a second operator $A_{0}$ such that the pair of operators $\left(A, A_{0}\right)$ satisfies assumptions (B.1)-(B.3), by

$$
\log Z=\frac{1}{2} \zeta^{\prime}\left(0 ; A, A_{0}\right)-\frac{1}{2} \zeta\left(0 ; A, A_{0}\right) \log \ell^{2}
$$

where $\ell$, a real non vanishing number, is the usual renormalization parameter.

Next, we recall the main result of [30] about the decomposition of the relative partition function of a finite temperature quantum field theory on an ultrastatic space time. Let $M$ be a smooth Riemannian manifold of dimension $n, n \in \mathbb{N}$, and consider the product $X=S_{\frac{\beta}{2 \pi}}^{1} \times M$, where $S_{r}^{1}$ is the circle of radius $r$. Let $\xi$ be a complex line bundle over $X$, and $L$ a self adjoint non negative linear operator in the Hilbert space $\mathcal{H}(M)$ of the $L^{2}$ sections of the restriction of $\xi$ onto $M$, with respect to some fixed metric $g$ on $M$. Let $H$ be the self adjoint non negative operator $H=-\partial_{u}^{2}+L$, in the Hilbert space $\mathcal{H}(X)$ of the $L^{2}$ sections of $\xi$, with respect to the product metric $d u^{2} \oplus g$ on $X$, and with periodic boundary conditions on the circle. Assume that there exists a second operator $L_{0}$ defined on $\mathcal{H}(M)$, such that the pair $\left(L, L_{0}\right)$ satisfies the previous assumptions (B.1)-(B.3). Then, by Lemma 2.2 of 30 , it follows that there exists a second operator $H_{0}$ defined in $\mathcal{H}(X)$, such that the pair $\left(H, H_{0}\right)$ satisfies those assumptions too. Under these requirements, we introduce the relative zeta regularized partition function of the model described by the pair of operators $\left(H, H_{0}\right)$ using equation (22), and we have the following result [30] Proposition 3.1.

Proposition IV.2 Let $L$ be a non negative self adjoint operator on $M$, and $H=-\partial_{u}^{2}+L$, on $S_{r}^{1} \times M$ as defined above. Assume there exists an operator $L_{0}$ such that the pair $\left(L, L_{0}\right)$ satisfies conditions (B.1)-(B.3). Then,

$$
\begin{aligned}
\zeta\left(0 ; H, H_{0}\right) & =-\beta \operatorname{Res}_{s=-\frac{1}{2}} \zeta\left(s ; L, L_{0}\right), \\
\zeta^{\prime}\left(0 ; H, H_{0}\right) & =-\beta \operatorname{Res}_{s=-\frac{1}{2}} \zeta\left(s ; L ; L_{0}\right)-2 \beta(1-\log 2) \operatorname{Res}_{s=-\frac{1}{2}} \zeta\left(s ; L, L_{0}\right)-2 \log \eta\left(\beta ; L, L_{0}\right),
\end{aligned}
$$

where $H_{0}=-\partial_{u}^{2}+L_{0}$, and the relative Dedekind eta function is defined by

$$
\log \eta\left(\tau ; L, L_{0}\right)=\int_{0}^{\infty} \log \left(1-\mathrm{e}^{-\tau v}\right) e\left(v ; L, L_{0}\right) d v .
$$

The residues and the integral are finite.

\section{B. Relative determinant for the Laplacian in the whole space}

The aim of this section, and of the following one, is to investigate the determinant and the partition function of the operator $-\Delta^{g_{R}}$ described in Section [II] by means of the technique described in the previous section. More precisely, we consider in this section the operator acting in the space $L^{2}\left(\mathbb{R}^{n}\right)$, and in the following section the operator acting in the half-space $L^{2}\left(\mathbb{H}^{n}\right)$. However, recalling the analysis of Section III, singular rank one perturbations of the Laplace operator on $L^{2}\left(\mathbb{R}^{n}\right)$ according to the definition given in [3] , are well defined only for $n \neq 2, n \leq 3$. As a consequence, in the following, we shall restrict ourselves to the cases $n=1$ and $n=3$. 
Remark IV.3 The operator $-\Delta^{g_{R}}$ in the three dimensional space was originally described in [5] and more recently in [1] Section 1.1, and in [3] Section 1.5. The operator $-\Delta^{g_{R}}$ in the one dimensional space was investigated in [1] Section I.3. In particular, it is worth to observe that the operators described by Albeverio 6 others are obtained as rank one singular perturbations of the maximal operator $-\Delta^{0}$ (in the language of [3] and Section [II) fixing the value of the free parameter $c$ by imposing the preservation of the symmetry under scaling transformations (as explained in Section III), while here we fix the value of the constant $c$ by the condition described at the end of Section III, namely $c=-\frac{b_{n}}{a_{n}}$. The two different prescriptions, however, define the same operator, as follows by comparing the formulas for the difference of the resolvents given in [1] Theorems I.1.1.2, and I.3.1.3, with the ones obtained here using Proposition [II.1, and Lemma II.17.

The relative zeta function and the relative partition function for the operator $-\Delta^{g_{R}}$ in $\mathbb{R}^{3}$ have been evaluated in Section 4.1 of [30], and the following results were obtained:

$$
\begin{aligned}
\zeta\left(s ;-\Delta^{g_{R}},-\Delta^{0}\right) & =\frac{1}{2} \frac{\left(2 \pi^{2} g_{R}\right)^{2 s}}{\cos \pi s} \\
\log \eta\left(\tau ;-\Delta^{g_{R}},-\Delta^{0}\right) & =\log \Gamma\left(\frac{\tau}{4 \pi^{3} g_{R}}\right)+\frac{1}{2} \log \frac{\tau}{4 \pi^{3} g_{R}}-\frac{\tau}{4 \pi^{3} g_{R}}\left(\log \frac{\tau}{4 \pi^{3} g_{R}}-1\right)-\frac{1}{2} \log 2 \pi \\
\log Z & =2\left(\log \frac{\ell}{2 \pi^{2} g_{R}}-1\right) \frac{\beta}{8 \pi^{3} g_{R}}-\log \eta\left(\beta ;-\Delta^{g_{R}},-\Delta^{0}\right)
\end{aligned}
$$

For completeness, we investigate here the one dimensional case. The trace of the difference of the resolvents is given in Corollary III.2, and using the results of Section IID (see also Remark III.3) we obtain

$$
r\left(\lambda ;-\Delta^{g_{R}},-\Delta^{0}\right)=\operatorname{Tr}\left(R\left(\lambda,-\Delta^{g_{R}}\right)-R\left(\lambda,-\Delta^{0}\right)\right)=-\frac{1}{2 \lambda(i b \sqrt{\lambda}+1)},
$$

where $b=\frac{1}{\pi g_{R}}$. The expansion for large $\lambda$ is

$$
r(\lambda)=\frac{1}{2 i b \lambda^{3 / 2}}+O\left(\frac{1}{\lambda^{2}}\right), \quad \operatorname{Im} \sqrt{\lambda}>0,
$$

and for small $\lambda$

$$
r(\lambda)=-\frac{1}{2 \lambda}+O(1)
$$

It follows that all the conditions (B.1)-(B.3) of Section IV.1 are satisfied with $\alpha_{0}=-\frac{3}{2}$ or $\alpha_{0}=-1$, and $\beta_{0}=-1$. However, it should be noted that since $\alpha_{0}=-\frac{3}{2}$ if $b \neq 0$, while $\alpha_{0}=-1$, if $b=0$, then condition (C) is satisfied when $b \neq 0$, but is not satisfied when $b=0$. This is consistent with the fact that the limit case $b=0$ gives $g_{R}=\infty$, that corresponds to the limit case of the Laplacian with Dirichlet boundary condition at $x=0$, and a relative zeta function can not be defined in this case.

Next, we evaluate the relative spectral measure

$$
e\left(v ; L, L_{0}\right)=\frac{v}{\pi i} \lim _{\epsilon \rightarrow 0^{+}}\left(r\left(v^{2} \mathrm{e}^{2 i \pi-i \epsilon} ; L, L_{0}\right)-r\left(v^{2} \mathrm{e}^{i \epsilon} ; L, L_{0}\right)\right) .
$$

We obtain (note that the spectral measure vanishes in both limit cases $b=0$ and $b=\infty$, corresponding to the Laplacian with Dirichlet boundary condition at $x=0$ and to the free Laplacian)

$$
e\left(v ;-\Delta^{g_{R}},-\Delta^{0}\right)=-\frac{b}{\pi\left(1+b^{2} v^{2}\right)},
$$

and a simple calculation using the formula for the zeta function given in Proposition IV.1 gives

$$
\zeta\left(s ;-\Delta^{g_{R}},-\Delta^{0}\right)=-\frac{\left(\pi g_{R}\right)^{-2 s}}{2 \sin (\pi s)} .
$$


Using the definition of the relative Dedekind eta function and equation (22) for the partition function, we also obtain

$$
\begin{aligned}
\log \eta\left(\tau ;-\Delta^{g_{R}},-\Delta^{0}\right) & =-\log \Gamma\left(\frac{g_{R}}{2} \tau\right)-\frac{1}{2} \log \frac{g_{R}}{2} \tau+\frac{g_{R}}{2} \tau\left(\log \frac{g_{R}}{2} \tau-1\right)+\frac{1}{2} \log 2 \pi \\
\log Z & =-\frac{\pi}{4} g_{R} \beta-\log \eta\left(\beta ;-\Delta^{g_{R}},-\Delta^{0}\right)
\end{aligned}
$$

\section{Relative determinant for the Laplacian in the half space}

We pass now to study the case of main interest, namely the operator $-\Delta^{g_{R}}$ acting in the space $L^{2}\left(\mathbb{H}^{n}\right), n=1$ and 3. The case $n=2$ presents non trivial technical aspects that we are not able to tackle at the moment, and therefore its investigation is postponed to a further occasion.

\section{The case $n=3$}

The operator $-\Delta^{g_{R}}$ in $\mathbb{H}^{3}$ is the operator with resolvent given in Proposition III.1 and corresponds to the Fourier transforms of the operator investigated in Section IIE. In order to apply the results of Section IV A. we first need to check the conditions (B.1)-(B.3). By Lemmas II.15 and III.4 the operator $-\Delta^{g_{R}}$ has pure continuous spectrum coinciding with the non negative real axis for all $g_{R} \geq 0$. We will restrict ourselves to this case. Consider the pair $\left(-\Delta^{g_{R}},-\Delta^{0}\right)$. By Corollary II.2 and Lemma II.23 (see also Remark III.3), the difference of the resolvent is of trace class with trace

$$
\begin{aligned}
r\left(\lambda ;-\Delta^{g_{R}},-\Delta^{0}\right)=\operatorname{Tr}\left(R\left(\lambda,-\Delta^{g_{R}}\right)-R\left(\lambda,-\Delta^{0}\right)\right) & =\frac{\pi^{2}\left(1-\mathrm{e}^{2 a i \sqrt{\lambda}}\right)}{2 i \sqrt{\lambda}\left(\frac{1}{g_{R}}-\frac{\pi^{2}}{2} i \sqrt{\lambda}+\frac{\pi^{2}}{4 a} \mathrm{e}^{2 i a \sqrt{\lambda}}\right)} \\
& =\frac{1-\mathrm{e}^{2 a i \sqrt{\lambda}}}{i \sqrt{\lambda}\left(b-i \sqrt{\lambda}+\frac{\mathrm{e}^{2 i a \sqrt{\lambda}}}{2 a}\right)},
\end{aligned}
$$

where $b=\frac{2}{\pi^{2} g_{R}}$, and $a$ is a real positive number that gives the position of the delta interaction. We obtain the expansions for large $|\lambda|$

$$
r(\lambda)=\frac{1}{\lambda}-\frac{i b}{\lambda^{3 / 2}}+O\left(\frac{1}{\lambda^{2}}\right), \quad \operatorname{Im} \sqrt{\lambda}>0
$$

and for small $|\lambda|$

$$
r(\lambda)=-\frac{4 a^{2}}{1+2 a b}-\frac{4 a^{3} i}{1+2 a b} \sqrt{\lambda}-\frac{16 a^{4}(1-a b)}{3(1+2 a b)^{2}} \lambda+O\left(\lambda^{3 / 2}\right),
$$

and this shows that all the conditions (B.1)-(B.3) of Section IVA are satisfied with $\alpha_{0}=-1<\beta_{0}=0$, and therefore also condition (C) is satisfied.

Second, we evaluate the relative spectral measure

$$
e\left(v ; L, L_{0}\right)=\frac{v}{\pi i} \lim _{\epsilon \rightarrow 0^{+}}\left(r\left(v^{2} \mathrm{e}^{2 i \pi-i \epsilon} ; L, L_{0}\right)-r\left(v^{2} \mathrm{e}^{i \epsilon} ; L, L_{0}\right)\right) .
$$

Substitution of the expression in equation (23) in equation (24) gives

$$
e\left(v ;-\Delta^{g_{R}},-\Delta^{0}\right)=-\frac{4 a}{\pi} \frac{1-2 a b+2 a b \cos (2 a v)-2 a v \sin (2 a v)-\cos (2 a v)}{1+4 a^{2}\left(b^{2}+v^{2}\right)+4 a b \cos (2 a v)-4 a v \sin (2 a v)} .
$$

The function $e\left(v ;-\Delta^{g_{R}},-\Delta^{0}\right)$ is a regular function of $v$ for all $v \geq 0$. For we show that there are no solution with $v \geq 0$ of the equation

$$
1+4 a^{2}\left(b^{2}+v^{2}\right)+4 a b \cos (2 a v)-4 a v \sin (2 a v)=0 .
$$


Consider the two curves:

$$
f_{1}(v)=4 a v \sin (2 a v)-4 a^{2} v^{2},
$$

and

$$
f_{2}(v)=1+4 a^{2} b^{2}+4 a b \cos (2 a v),
$$

and assume $a, b, v \geq 0$. Obviously, $f_{1}(v) \leq f_{3}(v)$ where $f_{3}(v)=4 a v-4 a^{2} v^{2}$, is a parabola facing down with vertex $V=\left(\frac{1}{2 a}, 1\right)$, that intercepts the horizontal axis in $v=0$ and $v=\frac{1}{a}$. On the other hand,

$$
(1+2 a b)^{2} \leq f_{2}(v) \leq(1-2 a b)^{2}
$$

and $f_{2}(v)$ oscillates around the value $1+4 a^{2} b^{2}$, and $f_{2}\left(\frac{\pi}{4 a}\right)=1+4 a^{2} b^{2}$. This suggests to split the problem into the three intervals $\left[0, \frac{\pi}{4 a}\right],\left[\frac{\pi}{4 a}, \frac{1}{a}\right]$, and $\left[\frac{1}{a}, \infty\right)$. In the interval $\left[0, \frac{\pi}{4 a}\right], f_{2}$ is decreasing and therefore

$$
f_{2}(v) \geq f_{2}\left(\frac{\pi}{4 a}\right)=1+4 a^{2} b^{2}
$$

On the other hand, in the same interval we have $f_{3}(v) \leq 1$ and therefore $f_{3}<f_{2}$ in this interval. In the interval $\left[\frac{1}{a}, \infty\right), f_{3}(v) \leq 0$, while $f_{2}(v) \geq(1-2 a b)^{2}$, and the value is zero if and only if $v=\frac{\pi(1+2 k)}{2 a}, k \in \mathbb{Z}$. But we have $f_{3}(v)=0$ if and only if $v=0$ or $v=\frac{1}{a}$, and $\frac{1}{a}<\frac{\pi}{2 a}$. Therefore, $f_{3}<f_{2}$ in this interval. Eventually, consider the interval $\left[\frac{\pi}{4 a}, \frac{1}{a}\right]$. In this interval, $f_{2}$ is decreasing and hence

$$
f_{2}(v) \geq f_{2}\left(\frac{1}{a}\right)=1+4 a^{2} b^{2}+4 a b \cos 2 .
$$

Also $f_{3}$ is decreasing, and hence

$$
f_{3}(v) \leq f_{2}\left(\frac{\pi}{4 a}\right)=\pi-\frac{\pi^{2}}{4}>0
$$

Now, we can check that $1+4 a^{2} b^{2}+4 a b \cos 2>\pi-\frac{\pi^{2}}{4}$, for all $a$ and $b$, hence $f_{3}<f_{2}$ on this interval, and this concludes the proof that $e\left(v ;-\Delta^{g_{R}},-\Delta^{0}\right)$ is a regular function of $v$ for all $v \geq 0$.

Third, we use Proposition IV.1 in order to obtain a suitable analytic extension of the relative zeta function. For we need the behavior for small and large $v$ of the function $e\left(v ;-\Delta^{g_{R}},-\Delta^{0}\right)$. We have

$$
e\left(v ;-\Delta^{g_{R}},-\Delta^{0}\right)=O\left(v^{2}\right)
$$

for $v \rightarrow 0^{+}$, and

$$
e\left(v ;-\Delta^{g_{R}},-\Delta^{0}\right)=\frac{2 \sin (2 a v)}{\pi v}+\frac{2 \sin ^{2}(2 a v)}{a \pi v^{2}}-\frac{2(1-2 a b) \sin ^{2}(a v)}{a \pi v^{2}}+O\left(v^{-3}\right),
$$

for $v \rightarrow+\infty$. So we decompose

$$
e\left(v ;-\Delta^{g_{R}},-\Delta^{0}\right)=e_{0}\left(v ;-\Delta^{g_{R}},-\Delta^{0}\right)+e_{\infty}\left(v ;-\Delta^{g_{R}},-\Delta^{0}\right),
$$

where

$$
\begin{aligned}
e_{\infty}\left(v ;-\Delta^{g_{R}},-\Delta^{0}\right) & =\frac{2 \sin (2 a v)}{\pi v}+\frac{2 \sin ^{2}(2 a v)}{a \pi v^{2}}-\frac{2(1-2 a b) \sin ^{2}(a v)}{a \pi v^{2}}, \\
e_{0}\left(v ;-\Delta^{g_{R}},-\Delta^{0}\right) & =e\left(v ;-\Delta^{g_{R}},-\Delta^{0}\right)-e_{\infty}\left(v ;-\Delta^{g_{R}},-\Delta^{0}\right)
\end{aligned}
$$

and

$$
\begin{aligned}
\zeta\left(s ;-\Delta^{g_{R}},-\Delta^{0}\right) & =\zeta_{0}\left(s ;-\Delta^{g_{R}},-\Delta^{0}\right)+\zeta_{\infty}\left(s ;-\Delta^{g_{R}},-\Delta^{0}\right) \\
& =\int_{0}^{\infty} v^{-2 s} e_{0}\left(v ;-\Delta^{g_{R}},-\Delta^{0}\right) d v+\int_{0}^{\infty} v^{-2 s} e_{\infty}\left(v ;-\Delta^{g_{R}},-\Delta^{0}\right) d v .
\end{aligned}
$$


Now $e_{0}\left(v ;-\Delta^{g_{R}},-\Delta^{0}\right)$ goes to a constant for $v \rightarrow 0$ and vanishes as $v^{-3}$ for $v \rightarrow \infty$, and so the function $\zeta_{0}\left(s ;-\Delta^{g_{R}},-\Delta^{0}\right)$ is a regular function of $s$ in the interval $-1<\operatorname{Re}(s)<\frac{1}{2}$. The function $\zeta_{\infty}\left(s ;-\Delta^{g_{R}},-\Delta^{0}\right)$ can be studied explicitly. We evaluate the integrals:

$$
\int_{0}^{\infty} v^{-2 s-1} \sin (2 a v) d v=-(2 a)^{2 s} \sin (\pi s) \Gamma(-2 s),
$$

for $-\frac{1}{2}<\operatorname{Re}(s)<\frac{1}{2}[18] 3.761 .4$

$$
\int_{0}^{\infty} v^{-2 s-2} \sin ^{2}(a v) d v=(2 a)^{2 s+1} \sin (\pi s) \Gamma(-2 s-1)
$$

for $-\frac{1}{2}<\operatorname{Re}(s)<\frac{1}{2}[18] 3.823$. Collecting, we have

$$
\zeta_{\infty}\left(s ;-\Delta^{g_{R}},-\Delta^{0}\right)=\frac{4}{\pi}(2 a)^{2 s} \sin (\pi s) \Gamma(-2 s-1)\left(a b+s+2^{2 s}\right) .
$$

Thus we have the following representation for the relative zeta function when $-\frac{1}{2}<\operatorname{Re}(s)<\frac{1}{2}$,

$$
\zeta\left(s ;-\Delta^{g_{R}},-\Delta^{0}\right)=\frac{4}{\pi}(2 a)^{2 s} \sin (\pi s) \Gamma(-2 s-1)\left(a b+s+2^{2 s}\right)+\int_{0}^{\infty} v^{-2 s} e_{0}\left(v ;-\Delta^{g_{R}},-\Delta^{0}\right) d v .
$$

This representation can be used in order to study the analytic continuation and in particular evaluate the residue and the finite part at $s=-\frac{1}{2}$. We obtain

$$
\begin{aligned}
\operatorname{Res}_{1} \zeta\left(s ;-\Delta^{g_{R}},-\Delta^{0}\right) & =\frac{2}{\pi^{2} g_{R}}, \\
\operatorname{Res}_{\substack{2 \\
s=-\frac{1}{2}}} \zeta\left(s ;-\Delta^{g_{R}},-\Delta^{0}\right) & =\frac{1+\log 2}{\pi a}+\frac{2 b(\gamma+\log (2 a))}{\pi}+\zeta_{0}\left(-\frac{1}{2} ;-\Delta^{g_{R}},-\Delta^{0}\right) \\
& =\frac{1+\log 2}{\pi a}+\frac{4(\gamma+\log (2 a))}{\pi^{2} g_{R}}+\int_{0}^{\infty} v e_{0}\left(v ;-\Delta^{g_{R}},-\Delta^{0}\right) d v .
\end{aligned}
$$

Using Proposition IV.2 and the formula in equation (22), we obtain the formula for the relative partition function

$$
\begin{aligned}
\log Z= & -\frac{\beta}{2} \operatorname{Res}_{s=-\frac{1}{2}} \zeta\left(s ;-\Delta^{g_{R}},-\Delta^{0}\right)-\beta(1-\log (2 \ell)) \operatorname{Res}_{s=-\frac{1}{2}} \zeta\left(s ;-\Delta^{g_{R}},-\Delta^{0}\right) \\
& -\log \eta\left(\beta ;-\Delta^{g_{R}},-\Delta^{0}\right) \\
= & \frac{2 \beta(\log (2 \ell)-1)}{\pi^{2} g_{R}}+\frac{\beta}{2}\left(\frac{1+\log 2}{\pi a}+\frac{4(\gamma+\log (2 a))}{\pi^{2} g_{R}}-\int_{0}^{\infty} v e_{0}\left(v ;-\Delta^{g_{R}},-\Delta^{0}\right) d v\right) \\
& -\log \eta\left(\beta ;-\Delta^{g_{R}},-\Delta^{0}\right) .
\end{aligned}
$$

As a consequence, the vacuum energy of the system reads

$$
\begin{aligned}
E_{c} & =-\lim _{\beta \rightarrow \infty} \frac{\partial}{\partial_{\beta}} \log Z \\
& =\frac{1+\log 2}{2 \pi a}+\frac{2}{\pi^{2} g_{R}}\left(\gamma+1+\log \frac{a}{\ell}\right)+\frac{1}{2} \int_{0}^{\infty} v e_{0}\left(v ;-\Delta^{g R},-\Delta^{0}\right) d v
\end{aligned}
$$

since for large $\beta$ the exponential in the integral dominates in the definition of $\log \eta\left(\beta ;-\Delta^{g_{R}},-\Delta^{0}\right)$.

We are interested in the behavior of the force $p=-\frac{\partial}{\partial a} E_{c}$ of the vacuum for small values of $a$. We need the expansion for small $a$ of the integral

$$
\begin{aligned}
\zeta_{0}\left(-\frac{1}{2} ;-\Delta^{g_{R}},-\Delta^{0}\right) & =\int_{0}^{\infty} v^{-2 s} e_{0}\left(v ;-\Delta^{g_{R}},-\Delta^{0}\right) d v \\
& =\frac{1}{a} \int_{0}^{\infty} \frac{x}{a} e_{0}\left(\frac{x}{a} ;-\Delta^{g_{R}},-\Delta^{0}\right) d x
\end{aligned}
$$


therefore we study the function

$$
f(x, a)=\frac{x}{a} e_{0}\left(\frac{x}{a} ;-\Delta^{g_{R}},-\Delta^{0}\right)=\frac{N(x, a)}{D(x, a)},
$$

where

$$
\begin{aligned}
N(x, a)= & -4 x \sin x\left(\cos (5 x)+4 a b \cos (3 x)+4 a^{2} b^{2} \cos x\right) \\
& -2 \sin x\left(2 a b \sin (5 x)+\left(1+8 a^{2} b^{2}\right) \sin (3 x)+4 a b\left(1-a b+2 a^{2} b^{2}\right) \sin x\right),
\end{aligned}
$$

and

$$
D(x, a)=\pi x\left(1+4 a^{2} b^{2}+4 x^{2}+4 a b \cos (2 x)-4 x \sin (2 x)\right)=\pi x g(x, a) .
$$

This shows that the integral

$$
\int_{0}^{\infty} f(x, a) d x=\sum_{i} \int_{0}^{\infty} \frac{N_{i}(x, a)}{D(x, a)} d x
$$

decomposes as a finite sum of terms, and in each term the numerator factors as

$$
N_{i}(x, a)=a^{p_{i}} h_{i}(x),
$$

where $p_{i}$ is $0,1,2$ or 3 , and the functions $h_{i}(x)$ are bounded. Thus, it remains to deal with the denominator. As a function of $a, g(x, a)$ is a parabola "facing up", so $g(x, a) \geq g\left(x, a_{0}\right)$, where $a_{0}$ is the vertex: so the solution of $\partial_{a} g(x, a)=8 b^{2} a+4 b \cos (2 x)=0$, i.e. $a_{0}=-\frac{\cos (2 x)}{2 b}$. Therefore

$$
D(x, a) \geq D\left(x, a_{0}\right)=\pi x\left(1+\frac{\cos ^{2}(2 x)}{2}+4 x^{2}-4 x \sin (2 x)\right)>0,
$$

where it is easy to see that the function $D\left(x, a_{0}\right)$ is positive for all $x$. Thus, $|D(x, a)|>\left|D\left(x, a_{0}\right)\right|$, and

$$
\int_{0}^{\infty}|f(x, a)| d x \leq \sum_{i} \int_{0}^{\infty} \frac{a^{p_{i}}\left|h_{i}(x)\right|}{\left|D\left(x, a_{0}\right)\right|} .
$$

Now, it is also easy to see that

$$
\int_{0}^{\infty} \frac{a^{p_{i}}\left|h_{i}(x)\right|}{\left|D\left(x, a_{0}\right)\right|}<\infty
$$

for all $i$, since $\frac{a^{p_{i}}\left|h_{i}(x)\right|}{\left|D\left(x, a_{0}\right)\right|} \sim \frac{1}{x^{2}}$ for each $i$. This proves that the integral

$$
\int_{0}^{\infty} \frac{x}{a} e_{0}\left(\frac{x}{a} ;-\Delta^{g_{R}},-\Delta^{0}\right) d x
$$

converges uniformly for $a$ in compact sets, and therefore we can evaluate the behavior for small $a$ taking the expansion of the integrand for small values of $a$. We obtain

$$
\zeta_{0}\left(-\frac{1}{2} ;-\Delta^{g_{R}},-\Delta^{0}\right)=\frac{1}{a}\left(I_{0}+a b I_{1}+a^{2} b^{2} I_{2}\right)+O\left(a^{2}\right) .
$$

The integrals $I_{n}$ can be performed numerically. In particular we have

$$
\begin{aligned}
& I_{0}=-\frac{2}{\pi} \int_{0}^{\infty} \frac{\sin u(\sin (3 u)+2 u \cos (5 u))}{u\left(1+4 u^{2}-4 u \sin (2 u)\right)} d u \sim-0.12, \\
& I_{1}=-\frac{4}{\pi} \int_{0}^{\infty} \frac{\sin u\left(4 u\left(1+4 u^{2}\right) \cos (3 u)-4 u \cos u-4 u^{2} \sin (5 u)+\left(1+16 u^{2}\right) \sin u\right)}{u\left(1+4 u^{2}-4 u \sin (2 u)\right)^{2}} \sim-0.51, \\
& I_{2}=-\frac{32}{\pi} \int_{0}^{\infty} \frac{u \sin u\left(8 u^{3} \cos u-6 u \cos (3 u)-12 u^{2} \sin u+\sin (5 u)\right)}{\left(1+4 u^{2}-4 u \sin (2 u)\right)^{3}} d u \sim-1.04 .
\end{aligned}
$$

This gives the behavior of the force for small $a$ :

$$
p=-\frac{\partial}{\partial a} E_{c}=\frac{1+\log 2+2 \pi I_{0}}{2 \pi a^{2}}-\frac{2}{\pi^{2} g_{R} a}-\frac{2 I_{2}}{\pi^{4} g_{R}^{2}}+O(a) .
$$

Using the numerical results given above, we see that for small values of $a$ the force is positive $\left(p \sim 0.15 / a^{2}\right)$. 


\section{The case $n=1$}

The operator $-\Delta^{g_{R}}$ in $\mathrm{H}^{1}=[0, \infty)$ is the operator with resolvent given in Proposition III.1 and corresponds to the Fourier transform of the operator investigated in Section IIE. By Lemmas II.15 and III.4, the operator $-\Delta^{g_{R}}$ has pure continuous spectrum coinciding with the non negative real axis if $a \leq \frac{2 g_{R}}{\pi}<\infty$. We will restrict our considerations to this case. Consider the pair $\left(-\Delta^{g_{R}},-\Delta^{0}\right)$. By Corollary II.2 and Lemma II.23 (see also Remark III.3), the difference of the resolvent is of trace class with trace

$$
r\left(\lambda ;-\Delta^{g_{R}},-\Delta^{0}\right)=\operatorname{Tr}\left(R\left(\lambda,-\Delta^{g_{R}}\right)-R\left(\lambda,-\Delta^{0}\right)\right)=-\frac{\pi}{4 i \lambda^{\frac{3}{2}}} \frac{1+\mathrm{e}^{2 a i \sqrt{\lambda}}(2 i a \sqrt{\lambda}-1)}{\frac{1}{g_{R}}+\frac{\pi}{4 i \sqrt{\lambda}}\left(1-\mathrm{e}^{2 i a \sqrt{\lambda}}\right)} .
$$

We obtain the expansions for large values of $|\lambda|$

$$
r\left(\lambda ;-\Delta^{g_{R}},-\Delta^{0}\right)=\frac{i g_{R} \pi}{4 \lambda^{3 / 2}}+O\left(\frac{1}{\lambda^{2}}\right), \quad \operatorname{Im} \sqrt{\lambda}>0,
$$

and for small values of $|\lambda|$

$$
r\left(\lambda ;-\Delta^{g_{R}},-\Delta^{0}\right)=\frac{i a^{2} g_{R} \pi}{\left(a g_{R} \pi-2\right) \sqrt{\lambda}}+\frac{a^{3} g_{R} \pi\left(a g_{R} \pi-8\right)}{3\left(a g_{R} \pi-2\right)^{2}}+O(\sqrt{\lambda}) .
$$

This shows that all the conditions (B.1)-(B.3) of Section IVA are satisfied with $\alpha_{0}=-3 / 2<\beta_{0}=-1 / 2$, and therefore also condition (C) is satisfied. Second, we evaluate the relative spectral measure (see definition in Proposition IV.1). We obtain

$$
e\left(v ;-\Delta^{g_{R}},-\Delta^{0}\right)=\frac{4 b \sin (a v)}{\pi} \frac{(a b+1) \sin (a v)-2 a v \cos (a v)}{b^{2}+2 v^{2}-b^{2} \cos (2 a v)-2 b v \sin (2 a v)},
$$

where we have set $b=\frac{\pi g_{R}}{2}$. Proceeding as in the previous section, we show that the function $e\left(v ;-\Delta^{g_{R}},-\Delta^{0}\right)$ is a regular function of $v$ for all $v \geq 0$. Third, we use Proposition IV.1 in order to obtain a suitable analytic extension of the relative zeta function. For we need the behavior for small and large values of $v$ of the function $e\left(v ;-\Delta^{g_{R}},-\Delta^{0}\right)$. We have

$$
e\left(v ;-\Delta^{g_{R}},-\Delta^{0}\right)=\frac{2 a^{2} b}{\pi(1-a b)}+O\left(v^{2}\right)
$$

for $v \rightarrow 0^{+}$, and

$$
e\left(v ;-\Delta^{g_{R}},-\Delta^{0}\right)=\frac{2 a b \sin (2 a v)}{\pi v}+\frac{2 b\left(a b \sin ^{2}(2 a v)-(1+a b) \sin ^{2}(a v)\right)}{\pi v^{2}}+O\left(v^{-3}\right),
$$

for $v \rightarrow+\infty$. So we decompose

$$
e\left(v ;-\Delta^{g_{R}},-\Delta^{0}\right)=e_{0}\left(v ;-\Delta^{g_{R}},-\Delta^{0}\right)+e_{\infty}\left(v ;-\Delta^{g_{R}},-\Delta^{0}\right),
$$

where

$$
\begin{aligned}
e_{\infty}\left(v ;-\Delta^{g_{R}},-\Delta^{0}\right) & =\frac{2 a b \sin (2 a v)}{\pi v}+\frac{2 b\left(a b \sin ^{2}(2 a v)-(1+a b) \sin ^{2}(a v)\right)}{\pi v^{2}} \\
e_{0}\left(v ;-\Delta^{g_{R}},-\Delta^{0}\right) & =e\left(v ;-\Delta^{g_{R}},-\Delta^{0}\right)-e_{\infty}\left(v ;-\Delta^{g_{R}},-\Delta^{0}\right)
\end{aligned}
$$

and

$$
\begin{aligned}
\zeta\left(s ;-\Delta^{g_{R}},-\Delta^{0}\right) & =\zeta_{0}\left(s ;-\Delta^{g_{R}},-\Delta^{0}\right)+\zeta_{\infty}\left(s ;-\Delta^{g_{R}},-\Delta^{0}\right) \\
& =\int_{0}^{\infty} v^{-2 s} e_{0}\left(v ;-\Delta^{g_{R}},-\Delta^{0}\right) d v+\int_{0}^{\infty} v^{-2 s} e_{\infty}\left(v ;-\Delta^{g_{R}},-\Delta^{0}\right) d v .
\end{aligned}
$$

As for the three-dimensional case, $e_{0}\left(v ;-\Delta^{g_{R}},-\Delta^{0}\right)$ is a constant at $v=0$ and goes to zero as $v^{-3}$ at infinity. Then $\zeta_{0}\left(s ;-\Delta^{g_{R}},-\Delta^{0}\right)$ is a regular function of $s$ in the interval $-1<\operatorname{Re}(s)<\frac{1}{2}$. 
The function $\zeta_{\infty}\left(s ;-\Delta^{g_{R}},-\Delta^{0}\right)$ can be studied explicitly. The integrals involved are of the same type of the ones evaluated in the previous section. For $-\frac{1}{2}<\operatorname{Re}(s)<\frac{1}{2}$, we obtain

$$
\zeta_{\infty}\left(s ;-\Delta^{g_{R}},-\Delta^{0}\right)=\frac{(2 a)^{2 s+1} b\left(2 s+\left(2^{2 s+1}-1\right) a b\right)}{\pi} \Gamma(-2 s-1) \sin (\pi s) .
$$

Thus we have the following representation for the relative zeta function when $-\frac{1}{2}<\operatorname{Re}(s)<\frac{1}{2}$,

$$
\begin{aligned}
\zeta\left(s ;-\Delta^{g_{R}},-\Delta^{0}\right)= & \frac{(2 a)^{2 s+1} b\left(2 s+\left(2^{2 s+1}-1\right) a b\right)}{\pi} \Gamma(-2 s-1) \sin (\pi s) \\
& +\int_{0}^{\infty} v^{-2 s} e_{0}\left(v ;-\Delta^{g_{R}},-\Delta^{0}\right) d v
\end{aligned}
$$

This representation can be used in order to study the analytic continuation and in particular evaluate the residue and the finite part at $s=-\frac{1}{2}$. We obtain

$$
\begin{aligned}
& \operatorname{Res}_{s=-\frac{1}{2}} \zeta\left(s ;-\Delta^{g_{R}},-\Delta^{0}\right)=-\frac{b}{2 \pi}=-\frac{g_{R}}{4}, \\
& \operatorname{Res}_{s=-\frac{1}{2}} \zeta\left(s ;-\Delta^{g_{R}},-\Delta^{0}\right)=\frac{b(1-\gamma-\log (2 a)+a b \log 2)}{\pi}+\zeta_{0}\left(-\frac{1}{2} ;-\Delta^{g_{R}},-\Delta^{0}\right) \\
& =\frac{g_{R}\left(2-\gamma-2 \log (2 a)+a g_{R} \pi \log 2\right)}{4}+\int_{0}^{\infty} v e_{0}\left(v ;-\Delta^{g_{R}},-\Delta^{0}\right) d v .
\end{aligned}
$$

Using Proposition IV.2 and the formula in equation (22), we obtain the formula for the relative partition function

$$
\begin{aligned}
\log Z= & -\frac{\beta}{2} \operatorname{Res}_{s=-\frac{1}{2}} \zeta\left(s ;-\Delta^{g_{R}},-\Delta^{0}\right)-\beta(1-\log (2 \ell)) \operatorname{Res}_{s=-\frac{1}{2}} \zeta\left(s ;-\Delta^{g_{R}},-\Delta^{0}\right) \\
& -\log \eta\left(\beta ;-\Delta^{g_{R}},-\Delta^{0}\right) \\
= & \frac{\beta g_{R}}{8}\left(2 \gamma-a g_{R} \pi \log 2+2 \log \frac{a}{\ell}\right)-\frac{\beta}{2} \int_{0}^{\infty} v e_{0}\left(v ;-\Delta^{g_{R}},-\Delta^{0}\right) d v \\
& -\log \eta\left(\beta ;-\Delta^{g_{R}},-\Delta^{0}\right) .
\end{aligned}
$$

As a consequence, the vacuum energy of the system reads

$$
\begin{aligned}
E_{c} & =-\lim _{\beta \rightarrow \infty} \frac{\partial}{\partial_{\beta}} \log Z \\
& =-\frac{g_{R}}{8}\left(2 \gamma-a g_{R} \pi \log 2+2 \log \frac{a}{\ell}\right)+\frac{1}{2} \int_{0}^{\infty} v e_{0}\left(v ;-\Delta^{g_{R}},-\Delta^{0}\right) d v
\end{aligned}
$$

since for large $\beta$ the exponential function dominates in the function $\log \eta\left(\beta ;-\Delta^{g_{R}},-\Delta^{0}\right)$.

We are interested in the behavior of the force $p=-\frac{\partial}{\partial a} E_{c}$ of the vacuum for small $a$. Therefore, we need an expansion for small values of $a$ of the integral

$$
\zeta_{0}\left(-\frac{1}{2} ;-\Delta^{g_{R}},-\Delta^{0}\right)=\int_{0}^{\infty} v e_{0}\left(v ;-\Delta^{g_{R}},-\Delta^{0}\right) d v
$$

Proceeding as in the previous section, we show that the integral converges uniformly on compact subsets. Expanding for small values of $a$ we obtain

$$
\zeta_{0}\left(-\frac{1}{2} ;-\Delta^{g_{R}},-\Delta^{0}\right)=-\frac{2 \log 2}{\pi} a b^{2}+O\left(a^{2}\right) .
$$

This gives the behavior of the force for small values of $a$ :

$$
p=-\frac{\partial}{\partial a} E_{c}=\frac{g_{R}}{4 a}+\frac{g_{R}^{2} \pi \log 2}{8}+O(a) .
$$

In this case the force is positive for small values of $a$. 


\section{APPENDIX}

Lemma V.1 Let $D_{N}^{n}$ be the closed disc of radius $N$ centered at the origin in $\mathbb{R}^{n}, n=1,2,3$. Then, for all $\lambda \in$ $\mathbb{C}-(-\infty, 0)$, and for large $N$

$$
\begin{aligned}
& \int_{D_{N}^{3}} \frac{1}{|x|^{2}-\lambda} d^{3} x=4 \pi N+2 \pi^{2} i \sqrt{\lambda} \operatorname{sgn}(\operatorname{Im} \sqrt{\lambda})+o(1), \\
& \int_{D_{N}^{2}} \frac{1}{|x|^{2}-\lambda} d^{2} x=2 \pi \log N-\pi \log (-\lambda)+o(1), \\
& \int_{D_{N}^{1}} \frac{1}{|x|^{2}-\lambda} d x=\frac{i \pi}{\sqrt{\lambda} \operatorname{sgn}(\operatorname{Im} \sqrt{\lambda})}-\frac{2}{N}+o\left(\frac{1}{N}\right) .
\end{aligned}
$$

Pooof. Using polar coordinates we have

$$
\begin{aligned}
\int_{D_{N}^{3}} \frac{1}{|x|^{2}-\lambda} d^{3} x & =4 \pi \int_{0}^{N} \frac{r^{2}}{r^{2}-\lambda} d r \\
& =4 \pi N+4 \pi \lambda \int_{0}^{N} \frac{d r}{r^{2}-\lambda}=4 \pi N+2 \pi^{2} i \sqrt{\lambda} \operatorname{sgn}(\operatorname{Im} \sqrt{\lambda})+o(1) .
\end{aligned}
$$

In a similar way we get corresponding results for $n=2$ and 1 .

Lemma V.2 For $n=1,2,3$ we have

$$
\int_{\mathbb{R}^{n}} \frac{d^{n} x}{\left.|| x\right|^{2} \pm\left. i\right|^{2}}= \begin{cases}\sqrt{2} \pi^{2}, & n=3 \\ \frac{\pi^{2}}{2}, & n=2 \\ \frac{\pi^{2}}{\sqrt{2}}, & n=1\end{cases}
$$

Pooof. We observe that

$$
\frac{1}{\left.|| x\right|^{2} \pm\left. i\right|^{2}}=\frac{1}{|x|^{4}+1}=\frac{1}{2 i}\left(\frac{1}{|x|^{2}+i}-\frac{1}{|x|^{2}-i}\right) .
$$

Then the results follow as a consequence of Lemma V.1.

Lemma V.3 Let $B_{N}^{n}$ be the closed half disc of radius $N$ centered at the origin in $\mathbb{R}^{n}, n=1,2,3$. Then, for all $\lambda \in \mathbb{C}-(-\infty, 0), a \in \mathbb{R}^{n}$ and large $N$

$$
\begin{aligned}
& \int_{B_{N}^{3}} \frac{\sin ^{2}(a x)}{|x|^{2}-\lambda} d^{3} x=\pi N+\frac{i \pi^{2}}{2} \sqrt{\lambda} \operatorname{sgn}(\operatorname{Im} \sqrt{\lambda})-\frac{\pi^{2}}{4 a} \mathrm{e}^{2 i a \sqrt{\lambda} \operatorname{sgn}(\operatorname{Im} \sqrt{\lambda})}+o(1), \\
& \int_{B_{N}^{2}} \frac{\sin ^{2}(a x)}{|x|^{2}-\lambda} d^{2} x=\frac{\pi}{2} \log N-\frac{\pi}{4} \log (-\lambda)+\frac{\pi}{2} K_{0}(2 a i \sqrt{\lambda})+o(1), \\
& \int_{B_{N}^{1}} \frac{\sin ^{2}(a x)}{|x|^{2}-\lambda} d x=\frac{i \pi}{4 \sqrt{\lambda} \operatorname{sgn}(\operatorname{Im} \sqrt{\lambda})}\left(1-\mathrm{e}^{2 i a \sqrt{\lambda} \operatorname{sgn}(\operatorname{Im} \sqrt{\lambda})}\right)-\frac{1}{2 N}+o\left(\frac{1}{N}\right),
\end{aligned}
$$

where $K_{0}(z)$ is a Bessel function.

Pooof. (See also [18] 3.723.3 and 3.723.10). First of all we observe that

$$
\int_{B_{N}^{n}} \frac{\sin ^{2}(a x)}{|x|^{2}-\lambda} d^{n} x=\frac{1}{2} \int_{D_{N}^{n}} \frac{\sin ^{2}(a x)}{|x|^{2}-\lambda} d^{n} x=\frac{1}{4} \int_{D_{N}^{n}} \frac{1}{|x|^{2}-\lambda} d^{n} x-\frac{1}{4} \int_{D_{N}^{n}} \frac{\cos (2 a x)}{|x|^{2}-\lambda} d^{n} x .
$$

Now we see that for $n=1,2,3$ the first integral in the latter line has been already computed in LemmaV.1, while for the second one, by taking polar coordinates and putting $a$ on the positive $z$-axis we get $(n=3)$

$$
\begin{aligned}
\int_{D_{N}^{3}} \frac{\cos (2 a x)}{|x|^{2}-\lambda} d^{2} x & =2 \pi \int_{r=0}^{N} \int_{u=-1}^{1} \frac{r^{2} \cos (2 a r u)}{r^{2}-\lambda} d u d r \\
& =\frac{2 \pi}{a} \int_{r=0}^{N} \frac{r \sin (2 a r)}{r^{2}-\lambda} d r=\frac{\pi^{2}}{a} e^{2 i a \sqrt{\lambda} \operatorname{sgn}(\operatorname{Im} \sqrt{\lambda})}+o(1),
\end{aligned}
$$


while for $n=2,1$ we obtain respectively

$$
\begin{gathered}
\int_{D_{N}^{2}} \frac{\cos (2 a x)}{|x|^{2}-\lambda} d^{2} x=-2 \pi K_{0}(2 a i \sqrt{\lambda})+o(1), \\
\int_{-N}^{N} \frac{\cos (2 a x)}{x^{2}-\lambda} d x=\frac{\pi i e^{2 i a \sqrt{\lambda} \operatorname{sgn}(\operatorname{Im} \sqrt{\lambda})}}{\sqrt{\lambda} \operatorname{sgn}(\operatorname{Im} \sqrt{\lambda})}+o(1 / N),
\end{gathered}
$$

$K_{0}(z)$ being a Bessel function. The thesis follows from these results and Lemma V.1.

By an easy computation we get:

Lemma V.4 For $n=1,2,3, a \in \mathbb{R}^{n}$ and $\operatorname{Im}(\sqrt{\lambda})>0$ we have

$$
\int_{\mathbb{R}^{n}} \frac{d^{n} x}{\left(|x|^{2}-\lambda\right)^{2}}= \begin{cases}\frac{i \pi^{2}}{\sqrt{\lambda}}, & n=3 \\ -\frac{\pi}{\lambda}, & n=2 \\ -\frac{i \pi}{2 \lambda^{3 / 2}}, & n=1 .\end{cases}
$$

Lemma V.5 For $n=1,2,3, a \in \mathbb{R}^{n}$ and $\operatorname{Im}(\sqrt{\lambda})>0$ we have

$$
\int_{\mathbb{R}^{n}} \frac{\sin ^{2}(a x)}{\left(|x|^{2}-\lambda\right)^{2}} d^{n} x= \begin{cases}\frac{i \pi^{2}}{2 \sqrt{\lambda}}\left(1-e^{2 i a \sqrt{\lambda}}\right), & n=3, \\ -\frac{\pi}{2 \lambda}-\frac{\pi i a}{\sqrt{\lambda}} K_{1}(-2 i a \sqrt{\lambda}), & n=2, \\ -\frac{i \pi}{4 \lambda^{3 / 2}}\left(1-\mathrm{e}^{2 i a \sqrt{\lambda}}\right)+\frac{\pi a}{2 \lambda} \mathrm{e}^{2 i a \sqrt{\lambda}}, & n=1 .\end{cases}
$$

where $K_{1}(z)$ is a Bessel function.

Pooof. As in Lemma $\mathrm{V.3}$ we write

$$
\int_{\mathbb{R}^{n}} \frac{\sin ^{2}(a x)}{\left(|x|^{2}-\lambda\right)^{2}} d^{n} x=\frac{1}{2} \int_{\mathbb{R}^{n}} \frac{1}{\left(|x|^{2}-\lambda\right)^{2}} d^{n} x-\frac{1}{2} \int_{\mathbb{R}^{n}} \frac{\cos (2 a x)}{\left(|x|^{2}-\lambda\right)^{2}} d^{n} x .
$$

Then, choosing polar coordinates and putting $a$ along the positive $z$-axis, for $n=3$ we get

$$
\begin{aligned}
\int_{\mathbb{R}^{3}} \frac{\cos (2 a x)}{\left(|x|^{2}-\lambda\right)^{2}} d^{3} x & =2 \pi \int_{r=0}^{\infty} \int_{u=-1}^{1} \frac{r^{2} \cos (2 a r u)}{\left(r^{2}-\lambda\right)^{2}} d u d r \\
& =\frac{2 \pi}{a} \int_{r=0}^{\infty} \frac{r \sin (2 a r)}{\left(r^{2}-\lambda\right)^{2}} d r=\frac{i \pi^{2}}{\sqrt{\lambda}} e^{2 i a \sqrt{\lambda}} .
\end{aligned}
$$

In a similar way we obtain

$$
\begin{aligned}
\int_{\mathbb{R}^{2}} \frac{\cos (2 a x)}{\left(|x|^{2}-\lambda\right)^{2}} d^{2} x & =\frac{2 \pi i a}{\sqrt{\lambda}} K_{1}(-2 i a \sqrt{\lambda}), \\
\int_{\mathbb{R}} \frac{\cos (2 a x)}{\left(x^{2}-\lambda\right)^{2}} d x & =-\left(\frac{\pi a}{\lambda}+\frac{i \pi}{2 \lambda^{3 / 2}}\right) \mathrm{e}^{2 i a \sqrt{\lambda}} .
\end{aligned}
$$

The thesis follows from these results and Lemma $\mathrm{V.4}$.

[1] S. Albeverio, F. Gesztesy, R. Hoegh-Krohn and H. Holden, Solvable Models in Quantum Mechanics, second edition, with an Appendix by P. Exner, AMS Chelsea Publishing, Providence 2005.

[2] S. Albeverio, Z. Brzeźniak and L. Dabrowski, Fundamental solution of the heat and Schrödinger equations with point interaction, J. Funct. Anal. 130 (1995) 220-254.

[3] S. Albeverio and P. Kurasov, Singular perturbations of differential operators, Cambridge University Press 2000.

[4] N.I. Akhiezer and I.M. Glazman, Theory of linear operators in Hilbert space, Frederik Ungar Pub. 1963.

[5] F.A. Berezin and L.D. Faddeev, A remark on Schröedinger Equation with a Singular Potential, Soviet Math. Dokl. 2 (1961) 372-375. 
[6] M.SH. Birman and D.R. Yafaev, The spectral shift function. The work of G.G. Krein and its further development, St. Petersburg Math. J. 4 (1993) 833-870.

[7] M. Bordag and D.V. Vassilevich, Heat kernel expansion for semitransparent boundaries, J. Phys. A 32 (1999) $8247-8259$.

[8] M. Bordag, I.G. Pirozhenko, V.V. Nesterenko, spectral analysis of a flat plasma sheet model, J. Phys. A 38 (2005) 11027.

[9] A.A. Bytsenko, G. Cognola, L. Vanzo and S. Zerbini, Quantum Fields and Extended Objects in Space-times with Constant Curvature Spatial Section, Phys. Rept. 266 (1996) 1-126.

[10] J.S. Dowker and G. Kennedy, Finite temperature and boundary effects in static space-times , J. Phys. A 11 (1978) 895.

[11] E. Elizalde, L. Vanzo and S. Zerbini, Zeta-function regularization, the multiplicative anomaly and the Wodzicki Residue, Comm. Math. Phys. 194 (1998) 613-630.

[12] A.A. Bytsenko, E. Elizalde, S. Odintsov, A. Romeo and S. Zerbini Zeta-function regularization with applications, World Scientific 1994.

[13] J. Brüning and R. Seeley, The resolvent expansion for second order regular singular operators, J. of Funct. An. 73 (1988) 369-415.

[14] G. Cognola, L. Vanzo and S. Zerbini, Vacuum energy in arbitrarily shaped cavities, J. Math. Phys. 33 (1992) $222-228$.

[15] G. Gibbons, Thermal zeta functions, Phys. Letters A 60 (1977) 385-386.

[16] P.B. Gilkey, K. Kirsten and D.V. Vassilevich, Heat trace asymptotics defined by transfer boundary conditions Lett. Math. Phys. 63 (2003) 29-37.

[17] N. Graham, R.L. Jaffe, V. Khemani, M. Quandt. M. Scandurra and H. Weigel Calculating vacuum energies in renormalizable quantum field theories: A New approach to the Casimir problem, Nucl. Phys. B 645 (2002) 49-84.

[18] I.S. Gradshteyn and I.M. Ryzhik, Table of integrals, series, and products, Academic Press 1994.

[19] S.W. Hawking, Zeta function regularization of path integral in curved spacetime, Comm. Math. Phys. 55 (1977) $133-148$.

[20] R.L. Jaffe and A. Scardicchio, Casimir buoyancy, JHEP 0506:006 (2006).

[21] N.R. Khusnutdinov, Zeta-function approach to Casimir energy with singular potentials, Phys. Rev. D 73 (2006) 025003.

[22] K.A. Milton, The Casimir Effect: The Physical Manifestation of Zero-Point Energy, World Scientific, Singapore 2001.

[23] V.M. Mostepanenko and N.N. Trunov, The Casimir Effect and its Applications, Oxford, Clarendon Press 1997.

[24] W. Müller, Relative zeta functions, relative determinants and scattering theory, CMP 192 (1998) 309-347.

[25] G. Ortenzi and M. Spreafico, Zeta function regularization for a scalar field in a compact domain, J. Phys. A 37 (2004) 11499-11517.

[26] D.K. Park, Green's function approach to two and three-dimensional delta function potentials and application to the spin $1 / 2$ Aharonov-Bohm problem, J. Math. Phys. 36 (1995) 5453-5464.

[27] D.B. Ray and I.M. Singer, R-torsion and the Laplacian on Riemannian manifolds, Adv. Math. 7 (1974) $145-210$.

[28] S. Scarlatti and A. Teta, Derivation of the time dependent propagator for the three-dimensional Schrödinger equation with one point interaction, J. Phys. A 23 (1990) L1033-L1035.

[29] S.N. Solodukhin, Exact solution for a quantum field with delta like interaction, Nucl. Phys. B 541 (1999) $461-482$.

[30] M. Spreafico and S. Zerbini, Finite temperature quantum field theory on non compact domains and application to delta interactions, Rep. Math. Phys. 63 (2009) 163-177.

[31] J. Weidman, Linear operators in Hilbert spaces, GTM 69, Springer-Verlag 1980. 